\title{
A Data Fusion Approach to Optimize Compositional Stability of Organic-inorganic Perovskites
}

Shijing Sun ${ }^{1 \dagger *}$, Armi Tiihonen ${ }^{1 \dagger}$, Felipe Oviedo ${ }^{1}$, Zhe Liu ${ }^{1}$, Janak Thapa ${ }^{1}$, Yicheng Zhao ${ }^{2,2 a}$, Noor Titan P. Hartono ${ }^{1}$, Anuj Goyal ${ }^{3}$, Thomas Heumueller ${ }^{2}$, ${ }^{2 a}$, Clio Batali ${ }^{1}$, Alex Encinas ${ }^{1}$, Jason J. Yoo $^{1}$, Ruipeng Li $^{4}$, Zekun Ren ${ }^{5}$, I. Marius Peters ${ }^{2}$, Christoph J. Brabec ${ }^{2,2 a}$, Moungi G. Bawendi ${ }^{1}$, Vladan Stevanovic ${ }^{3}$, John Fisher III ${ }^{1}$, Tonio Buonassisi ${ }^{1,5 *}$

\author{
${ }^{1}$ Massachusetts Institute of Technology, Cambridge, MA 02139, USA \\ ${ }^{2}$ Helmholtz-Institute Erlangen-Nürnberg (HI-ERN), Erlangen, 91058, Germany \\ ${ }^{2 a}$ Institute of Materials for Electronics and Energy Technology (i-MEET), FAU, Erlangen, \\ 91058, Germany \\ ${ }^{3}$ Colorado School of Mines, Golden, CO 80401, USA \\ ${ }^{4}$ Brookhaven National Laboratory, Upton, NY 11970, USA \\ ${ }^{5}$ Singapore-MIT Alliance for Research and Technology, 138602, Singapore
}

†These authors contribute equally.

*Correspondence to: shijings@ mit.edu, buonassisi@mit.edu 


\section{Summary}

An outstanding challenge in creating environmentally stable perovskite semiconductors is the resource-efficient materials search in vast compositional spaces. Here we demonstrate a physicsconstrained sequential learning framework to autonomously identify the most stable alloyed organic-inorganic perovskites. We fuse data from high-throughput degradation tests and firstprinciple calculations of phase thermodynamics into an end-to-end Bayesian optimisation algorithm using probabilistic constraints. By sampling just $1.8 \%$ of the discretized $\mathrm{Cs}_{\mathrm{x}} \mathrm{MA}_{\mathrm{y}} \mathrm{FA}_{1-\mathrm{x}-}$ ${ }_{\mathrm{y}} \mathrm{PbI}_{3}(\mathrm{MA}=$ methylammonium, $\mathrm{FA}=$ formamidinium $)$ compositional space, multi-cation perovskites centred at $\mathrm{Cs}_{0.17} \mathrm{MA}_{0.03} \mathrm{FA}_{0.80} \mathrm{PbI}_{3}$ are identified showing minimal optical change under elevated temperature, moisture, and illumination with $>17 \times$ stability improvement from $\mathrm{MAPbI}_{3}$. This results in thin-films with $3 \times$ improved stability compared to state-of-the-art multi-halide $\mathrm{Cs}_{0.05}\left(\mathrm{MA}_{0.17} \mathrm{FA}_{0.83}\right)_{0.95} \mathrm{~Pb}\left(\mathrm{I}_{0.83} \mathrm{Br}_{0.17}\right)_{3}$, translating into enhanced solar cell stability under damp heat tests without compromising conversion efficiency. Synchrotron-based X-ray scattering validates the effective suppression of chemical decomposition and minority phase formation achieved using fewer elements and $8 \%$ or less MA. We anticipate that this data fusion approach can be extended to guide materials discovery for a wide range of multinary systems. 


\section{Introduction}

The environmental instability of organic-inorganic halide perovskite materials limits their usage in optoelectronics such as solar cells, light emitters, lasers, and photodetectors. ${ }^{1}$ Compositional engineering is to date one of the most effective methods to improve perovskites' stability in the presence of heat, humidity and light without sacrificing optoelectronic performance. ${ }^{2}$ This fact has led to intensive research within combinatorial spaces such as $A_{\mathrm{x}} B_{\mathrm{y}} C_{1-\mathrm{x}-\mathrm{y}} \mathrm{Pb}\left(\mathrm{I}_{\mathrm{z}} \mathrm{Br}_{1-\mathrm{z}}\right)_{3}{ }^{3}{ }^{3}$ However, only a small fraction of this compositional space has been experimentally explored, in part due to the prohibitively expensive brute force synthesis. The paucity of resulting degradation data inhibits generalisation of mechanisms across this diverse chemical and structural space, requiring each compositional search to start their experimental investigations ab initio. ${ }^{4}$ This challenge is similar to those faced by other materials communities, including heterogeneous catalysts, alloyed battery electrodes, and high-entropy metal alloys for structural and magnetic materials. ${ }^{5-7}$ The halide perovskite field and several others require new tools to experimentally navigate these vast spaces efficiently to locate optima and to extract generalisable scientific insights. ${ }^{8-14}$

Machine-learning-based sequential learning approaches (e.g. Bayesian optimisation, BO) have emerged as efficient materials search tools that explore vast variable spaces in a 'closed-loop' fashion, whereby the outcome of one experimental round informs the next without human intervention. BO has attracted increasing attention in the recent developments of self-driving laboratories in various fields of materials science, recently successfully directed high-throughput experimentation in the search of organic hole-transport materials ${ }^{15}$, piezoelectric oxides, ${ }^{16}$ and organic photocatalysts. ${ }^{10}$ However, such model-free statistical approach show limitations without principled guidance from domain expertise, because it has to learn everything from scratch. In the field of perovskite optoelectronics, recent in situ experiments and first-principle calculations independently reveal insights into the fundamental composition-dependent instability in organicinorganic perovskites and their alloys, however, merging computational and experimental insights on selective compositions into a generalisable optimisation policy over the entire chemical space remains a challenge. ${ }^{3}$ State-of-the-art two-step approaches of directly applying theoretical screening as a hard constraint prior to shortlisted synthesis are limited by the inefficiencies from: 1) high-performing theoretical calculations for organic-inorganic systems are often too sparse to guide experimentation, and 2) the discrepancies between the calculation assumptions and the 
experiments at non-thermodynamic equilibria decreases search accuracy. ${ }^{17,18}$ The lack of physicsinformed and iterative materials search hinders the ultimate goal of designing perovskite compositions for enhanced environmental stability.

Here we introduce a data fusion approach to incorporate both Gibbs free energy of mixing $\left(\Delta G_{\text {mix }}\right)$ from density functional theory (DFT) calculations ${ }^{19}$ and experimentally quantified degradation from accelerated aging tests to every decision that the $\mathrm{BO}$ algorithm is making. We apply this closed-loop machine-learning framework to optimise lead-iodide perovskites that suffer from severe heat and moisture-induced degradation within the five-element space of $\mathrm{Cs}_{\mathrm{x}} \mathrm{MA}_{\mathrm{y}} \mathrm{FA}_{1-\mathrm{x}-\mathrm{y}} \mathrm{PbI}_{3}$. Under multiplex environmental stress tests with elevated temperature, humidity, and illumination in air, we identify compositions overperforming the $\mathrm{MAPbI}_{3}$ starting-point by $17 \times$ and our stateof-the-art reference composition of $\left(\mathrm{Cs}_{0.05}\left(\mathrm{MA}_{0.17} \mathrm{FA}_{0.83}\right)_{0.95} \mathrm{~Pb}\left(\mathrm{I}_{0.83} \mathrm{Br}_{0.17}\right)_{3}\right)$ by $3 \times$ within three optimisation rounds, and the results are found transferable to device stability. DFT here serves as principled guidance within the decision-making algorithm to constrain the search space to not only chemically, but also the structurally stable $\alpha$-perovskite alloys.

\section{Results and Discussion}

Closed-loop Experimentation Platform Driven by Physics-informed Bayesian Optimisation 
a. Nominal compositions

b. Modelled phase stability

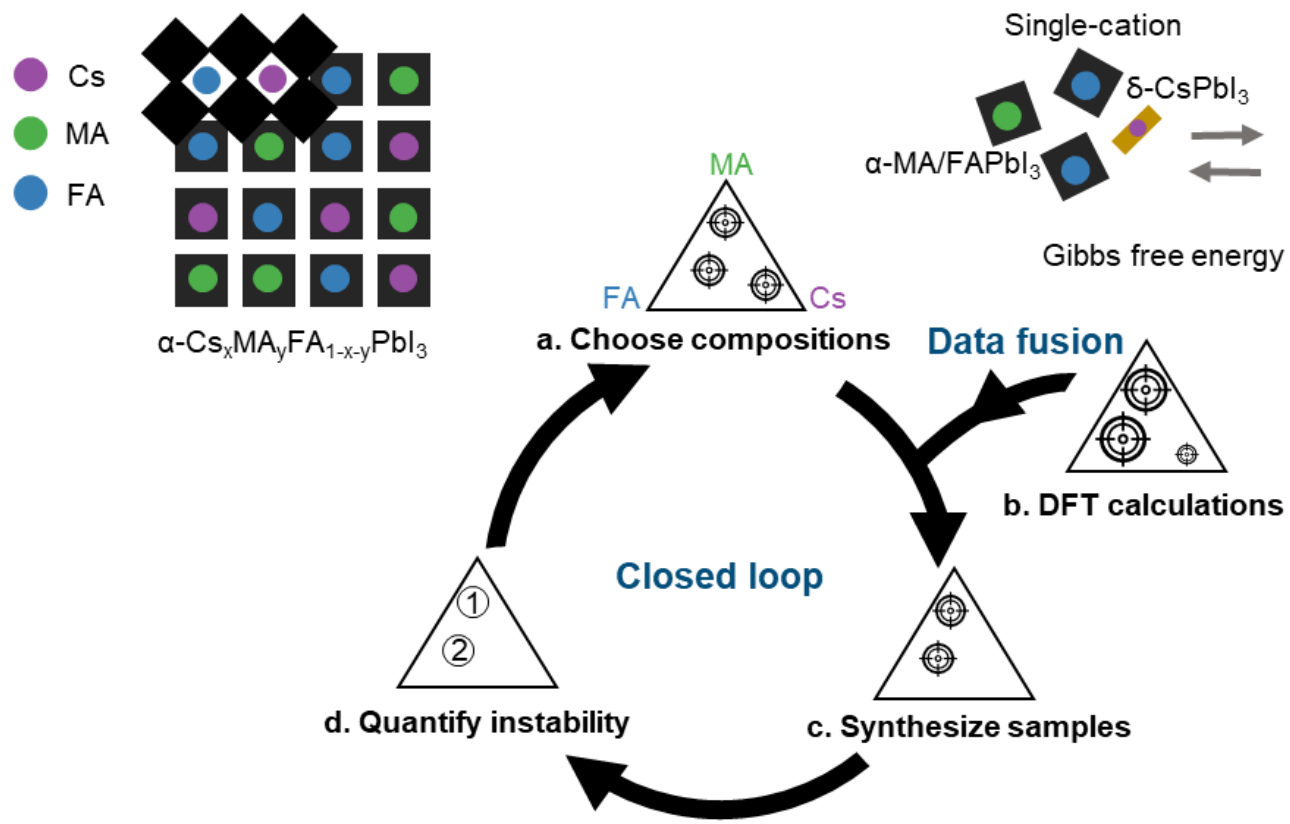

d. Degradation in hot and humid conditions

c. Phase mixing and demixing
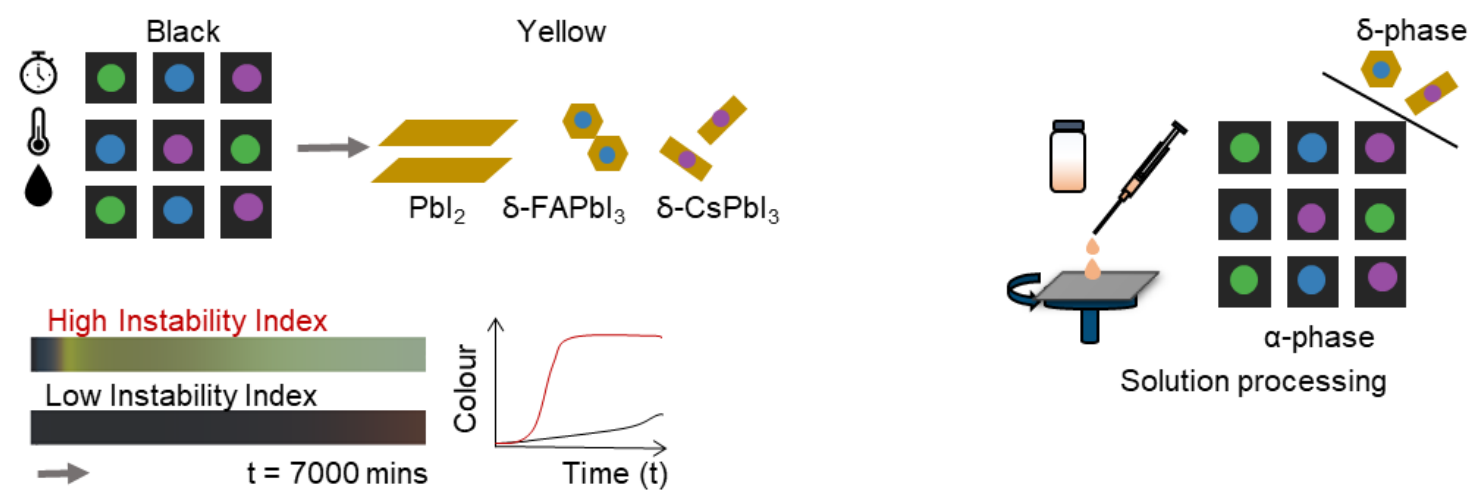

$\rightarrow \quad t=7000$ mins

Time $(\mathrm{t})$

Time-resolved sample photographs

Figure 1 Each optimisation round consists of three steps of 'composition selection', 'film synthesis', and 'instability quantification' and a fourth step of theoretical incorporation into the closed-loop workflow by a data fusion approach. a-b. Composition selection: the selection of nominal compositions of multi-cation perovskites is driven by machine learning algorithms with DFT-modelled $\Delta G_{m i x}$ incorporated by data fusion. c. Film synthesis: tuning A-site cations in lead iodide perovskites forms $\mathrm{Cs}_{\mathrm{x}} \mathrm{MA}_{\mathrm{y}} \mathrm{FA}_{1-\mathrm{x}-\mathrm{y}} \mathrm{PbI}_{3}$, a space of 5151 compositions (estimated experimental resolution 1\%). Thin-film samples are spin-coated in series using precursor solutions of nominal compositions. d. Instability quantification: we perform accelerated degradation tests with in situ optical monitoring, enabling 28 thinfilm samples being degraded in parallel. Near-black photoactive perovskite films turn yellow over time due to the emergence of high-bandgap degradation products. Quantified optical changes over time, recorded in 
R (red), G (green), and B (blue) channels, are used as a proxy to evaluate the chemical instability of the samples under $85 \%$ relative humidity $(\mathrm{RH}), 85^{\circ} \mathrm{C}$ sample temperature, and 0.15 Sun visible only illumination. Two representative sets of sample photographs and curves of the total $(R+G+B)$ areaaveraged value as a function of time are illustrated in $\mathrm{d}$.

To efficiently guide the compositional search, we construct a physics-informed batch Bayesian optimisation (BO) framework (Figure 1). In BO, promising compositions for the next experimental round are suggested by an acquisition function, such as expected improvement $E I(\Theta)$ that balances the exploitation of the most stable regions and the exploration of high-uncertainty regions within the compositional space. As a key algorithm contribution, we fuse $\Delta G_{m i x}$ as a probabilistic constraint of the BO acquisition function in the 'composition selection' step, providing additional information on phase stability to effectively identify multi-cation perovskites that are thermodynamically stable relative to their single-cation counterparts (Figure 1a-b). We define 'Instability Index' $\left(I_{c}\right)$, a figure of merit for optimising stability. The goal of each optimisation round, which consists of three steps of 'composition selection', 'film synthesis' and 'instability quantification', is to minimise this value. Our batch BO algorithm makes use of a surrogate ML model, Gaussian process regression $(\mathrm{GP}),{ }^{20}$ to estimate the value and uncertainty of $I_{c}$ in nonexplored regions of the compositional space (see Experimental Procedures).

Within each optimisation round (one batch in BO), 28 spin-coated thin-film samples (Figure 1c) are examined in situ in parallel using an environmental chamber under $85 \mathrm{RH} \%$ and $85^{\circ} \mathrm{C}$ in the air (Figure S1). 0.15 Sun visible only illumination is applied to enable automatic image capture every five minutes by an RGB camera ( $200 \mu \mathrm{m}$ resolution). Photoactive $\alpha$-perovskite phases within $\mathrm{Cs}_{\mathrm{x}} \mathrm{MA}_{\mathrm{y}} \mathrm{FA}_{1-\mathrm{x}-\mathrm{y}} \mathrm{PbI}_{3}$ exhibit a bandgap of $\sim 1.5 \mathrm{eV}$, whereas their main degradation products under hot and humid conditions, $\mathrm{PbI}_{2}(2.27 \mathrm{eV}),{ }^{21} \delta-\mathrm{CsPbI}_{3}(2.82 \mathrm{eV})^{22}$ or $\delta-\mathrm{FAPbI}_{3}(2.43 \mathrm{eV})^{23}$ show deteriorated photophysical properties (Figure S2). As per Figure 1d, we hence employ a colour-based metric as a proxy to capture the macroscopic evolution of the high-bandgap, nonperovskite phases. ${ }^{24-26}$ We define Instability Index $\left(I_{c}\right)$ as the integrated colour change of an unencapsulated perovskite film over accelerated degradation test duration $T$.

$$
I_{c}(\Theta)=\sum_{c=\{R, G, B\}} \int_{0 \min }^{T}|c(t, \Theta)-c(0, \Theta)| d t
$$


where composition $\Theta=(x, y, 1-x-y), t$ is time, and $c$ are area-averaged, colour-calibrated red, green and blue pixel values of the sample. The cut-off time was set to $T=7000 \mathrm{~min}$ based on the observed divergence between the most and least stable compositions (Figure S3). Our closed-loop and iterative workflow enable the systematic optimisation of multi-cation perovskites against degradation by varying the nominal compositions, $\Theta$, within $\mathrm{Cs}_{\mathrm{x}} \mathrm{MA}_{\mathrm{y}} \mathrm{FA}_{1-\mathrm{x}-\mathrm{y}} \mathrm{PbI}_{3}(x, y$ limit to two decimal places) (Table S1-2).

\section{Data fusion Approach: Incorporation of Phase Thermodynamics into Automated Composition}

\section{Selection}

Due to their polymorphic nature, identical perovskite compositions crystallised into different phases can exhibit diverse degradation behaviours, making it essential to evaluate phase stabilities in any perovskite composition optimisation. ${ }^{1}$ The end members of the compositional space in this study consist of the cubic $\alpha-\mathrm{FA} / \mathrm{MAPbI}_{3}$ perovskites and the non-perovskite $\delta-\mathrm{CsPI}_{3}$ at the synthesis temperature. ${ }^{27}$. Phase de-mixing during synthesis leads to minority phases within thinfilm samples prior to degradation tests and are, therefore, not captured in $I_{c}$. Schelhas et al. recently demonstrated the use of DFT calculations to predict the phase de-mixing tendency between $\alpha$ $\mathrm{Cs}_{\mathrm{x}} \mathrm{MA}_{\mathrm{y}} \mathrm{A}_{1-\mathrm{x}-\mathrm{y}} \mathrm{PbI}_{3}\left(G_{m i x}\right)$ and their single-cation perovskite polymorphs $A \mathrm{PbI}_{3}(A=\mathrm{Cs}, \mathrm{MA}$, or FA) $\left(G_{0}\right)$ at a given temperature. ${ }^{19}$ We herein fuse the composition-dependent change in Gibbs free energy of mixing, $\Delta G_{m i x}$ as a constraint into the experimental optimisation loop (Figure 2a). This approach allows the $\alpha$ - and $\delta$-phase relative stability to be considered in the composition selection, thus enabling us to reduce sampling in regions with high probability of minority phase formation. 

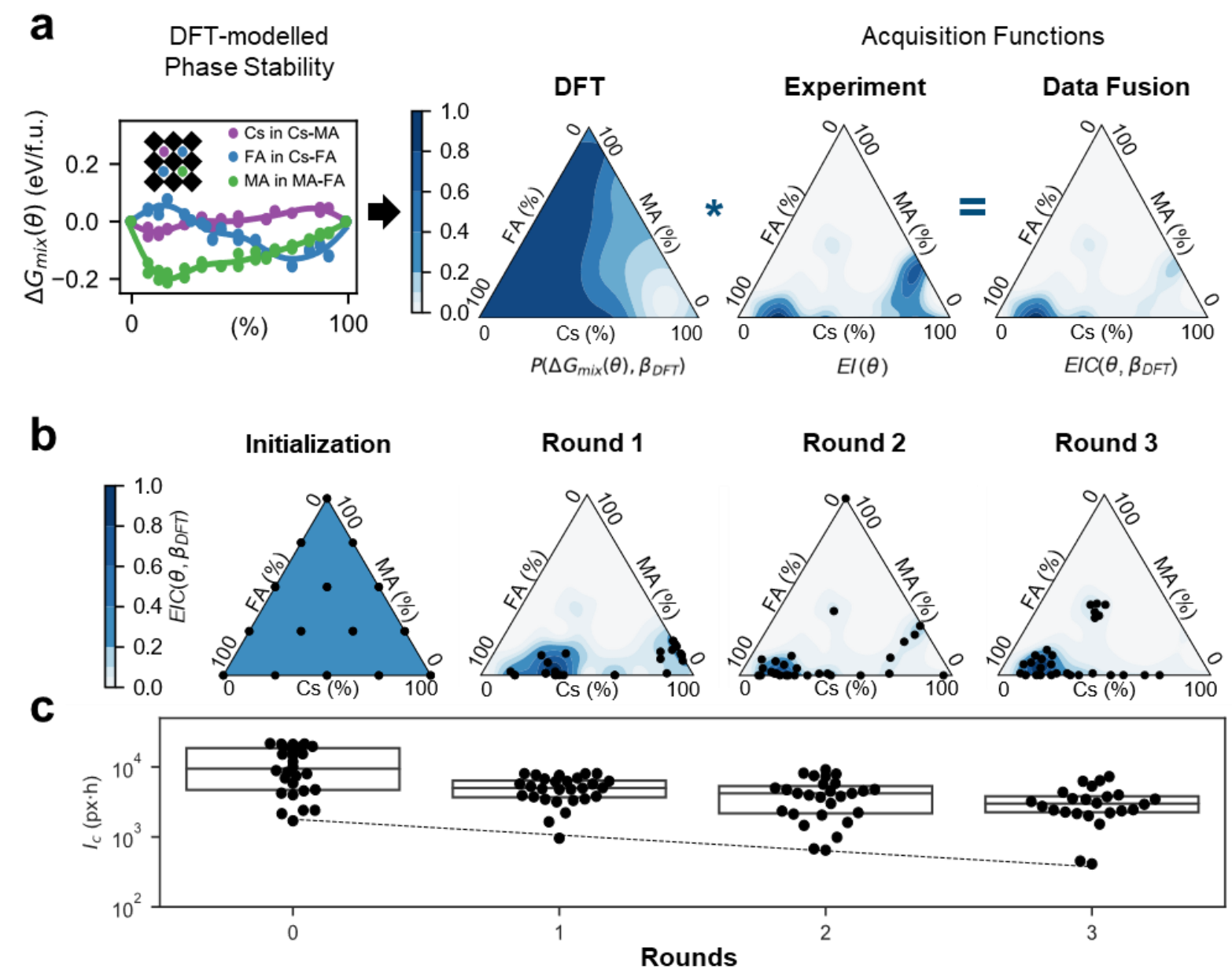

Figure 2 Fusion of DFT and experiments guides the optimisation of compositional stability. a. DFTmodelled Gibbs free energy of mixing, $\Delta G_{m i x}$, of 47 binary compositions of CsMA, CsFA, and MAFA $\alpha$ perovskites relative to end members of $\delta-\mathrm{CsPI}_{3}, \alpha-\mathrm{MAPbI}$, and $\alpha-\mathrm{FAPbI}_{3}$. We fit a Gibbs free-energy model, $\Delta G_{\text {mix }}(\Theta)$, where $\Theta$ is a composition in the ternary space, to the phase thermodynamics data using Gaussian process regression. $\Delta G_{m i x}(\Theta)$ is transformed into a probabilistic constraint, $P\left(\Delta G_{m i x}(\Theta), \beta_{D F T}\right)$ that models the cumulative probability of phase mixing at above $300 \mathrm{~K}$. Multiplying $P$ with the acquisition function of the Bayesian optimisation algorithm, $E I(\Theta)$, gives a DFT-weighted acquisition function, $E I C(\Theta)$. b. Starting from equally-spaced 15 compositions in the initialization round, in each optimisation round, 28 sample films (black markers) are synthesized and undergo degradation tests. Compositions are chosen by Bayesian optimisation algorithm that suggests them using $\operatorname{EIC}(\Theta)$ (blue surface colour). c. Experimentally measured Instability Indices, $I_{c}$ (pixels*hours), of 112 samples over one initialisation and three optimisation rounds. The black boxes indicate the mean and standard deviation of each round. The dashed line indicating the most stable compositions in each experimental round is for eye guidance only. 
Data fusion refers to a set of techniques where ML is used to map two or more datasets coming from related but distinct distributions. In our case, we relate the theoretical $\Delta G_{m i x}(\Theta)$ and the experimental $I_{c}(\Theta)$. The two data streams account for distinct mechanisms of modelled thermodynamic phase instability and measured macroscopic thermal-moisture instability, respectively. Hence, it is inadequate to combine both datasets as equivalent or include DFT directly as a prior following state-of-the-art model-free $\mathrm{BO} .{ }^{28,29} \mathrm{We}$ herein define a data-fused probabilistic constraint approach according to Eq. (2):

$$
P\left(\Delta G_{m i x}(\Theta), \beta_{D F T}\right)=\frac{1}{1+e^{-\Delta G_{m i x}(\Theta) / \beta_{D F T}}}
$$

where $P\left(\Delta G_{\text {mix }}(\Theta), \beta_{D F T}\right)$ is a logistic cumulative distribution function (CDF) modelling the phase mixing probability and $\beta_{D F T}$ is a data fusion parameter calibrated according to $\Delta G_{m i x}$ calculations to control the smoothness of the boundaries from stable to unstable compositions, forming a soft compositional boundary presented in Figure 2a (see Experimental Procedures for algorithm details).

Given the computational cost and complexity of DFT calculations on organic-inorganic hybrid systems, we first regress 85 DFT-modelled $\Delta G_{\text {mix }}$ values on 47 single-cation and binary alloyed compositions (29 MAFA and CsFA compositions from Schelhas et al. ${ }^{19} 12$ CsMA compositions computed for the present work using the same method) over the quasi-ternary $\mathrm{Cs}_{\mathrm{x}} \mathrm{MA}_{\mathrm{y}} \mathrm{A}_{1-\mathrm{x}-\mathrm{y}} \mathrm{PbI}_{3}$ phase space using an auxiliary GP model that defines $\Delta G_{m i x}(\Theta)$. Figure 2a visualises the probability of phase mixing $P\left(\Delta G_{m i x}(\Theta), \beta_{D F T}\right) \in[0,1]$, where low values suggest phase instability $\left(\Delta G_{m i x}>>0\right)$ and high values suggest phase stability $\left(\Delta G_{m i x}<<0\right)$.

Our work is inspired by the unknown constraint $\mathrm{BO}$ proposed by Gelbart et al. ${ }^{30} \mathrm{By}$ developing a probabilistic constraint model $P\left(\Delta G_{m i x}(\Theta), \beta_{D F T}\right)$ instead of applying a hard-constraint boundary, we are able to discount regions predicted by DFT to go through phase demixing rather than completely exclude any unfavourable regions. This approach accounts for the inherent uncertainty in DFT predictions, chemical accuracy, and data scarcity through the use of the soft compositional boundary to model the stability threshold (see Experimental Procedures for $\beta_{D F T}$ calibration). The proposed algorithm allows us to seamlessly adapt DFT into the experimental optimisations loop, 
thereby achieving a physics-informed and sample-efficient search without being limited by the unknown exact phase boundaries across a vast compositional space (Figure S4-5).

To integrate the probabilistic constraint into the $\mathrm{BO}$ formulation, we weigh the acquisition function with the value of $P\left(\Delta G_{m i x}(\Theta), \beta_{D F T}\right)$ and obtain a DFT-weighted BO acquisition function, $E I C(\Theta)$, as illustrated in Figure 2a. Traditional $E I(\Theta)$ utilizes the $I_{c}$ results of our first experimental round without DFT and indicates two potential optima in Cs-poor and Cs-rich regions, respectively. The DFT-weighted $\operatorname{EIC}(\Theta)$ effectively reduces sampling in energetically unfavourable Cs-rich regions despite low $I_{C}$ : the subsequent optimisation rounds converge to stable nominal compositions with a high probability of stable $\alpha$-perovskite films among Cs-poor regions (Figure 2a, Figure S6-7). Comparisons of optimisation with and without DFT-weighting using a teacher-student model are shown in Figure S8-9, which validates that without data fusion, the model-free BO algorithm continues to suggest sampling in Cs-rich regions despite of their phase instability.

Figure $2 \mathrm{~b}$ demonstrates that batch $\mathrm{BO}$ sequentially identifies the most stable regions over one initialization and three optimisation rounds of synthesis and degradation tests. Iterative evolution of the landscape (posterior mean of $I_{c}, I_{c}(\Theta)$, with uncertainty) is presented in Figure S3-S4. Figure $2 \mathrm{c}$ reveals a rapid decrease in experimentally quantified $I_{c}$ from Rounds $0-3$. The search converges after three optimisation rounds (see Figure S5 for convergence conditions) to an optimal composition region centred at $\mathrm{Cs}_{0.17} \mathrm{MA}_{0.03} \mathrm{FA}_{0.80} \mathrm{PbI}_{3}$ and bounded by $8-29 \% \mathrm{Cs},<14 \% \mathrm{MA}$ and 68-92 \% FA. The identification of the global optimum lying within an FA-rich, Cs and MA-poor region is consistent with the reports that FA-rich perovskites show superior environmental stability compared with their MA-rich counterparts and the less volatile Cs is expected to enhance the heat and moisture resistance. ${ }^{31}$ Interestingly, we found a local optimum near $\mathrm{Cs}_{0.26} \mathrm{MA}_{0.36} \mathrm{FA}_{0.38} \mathrm{PbI}_{3}$, which emerged in Round 1. We intentionally sample four additional compositions and verify the existence of local optima in Round 3. We define the compositional space as the discretized quasiternary phase space subdivided by the minimum achievable experimental resolution (1\% composition). This yields 5151 possible singular, binary and ternary cation compositions, $1.8 \%$ of which were sampled experimentally while converging to the optimal region. Three additional degradation rounds of seven representative compositions were performed, to validate the instability trend with structural and optical characterisation shown in the Table S3, Figure S10-13. 
Composition-, Phase-, and Time-dependent Instability Landscapes

We find the overall stability landscape within the $\mathrm{Cs}_{\mathrm{x}} \mathrm{MA}_{\mathrm{y}} \mathrm{FA}_{1-\mathrm{x}-\mathrm{y}} \mathrm{PbI}_{3}$ compositional space to be non-linear. To quantify the divergence in degradation profiles, Figure 3a presents the compositiondependent instability landscape, $I_{c}(\Theta)$ for $\mathrm{Cs}_{\mathrm{x}} \mathrm{MA}_{\mathrm{y}} \mathrm{FA}_{1-\mathrm{x}-\mathrm{y}} \mathrm{PbI}_{3}$ after three experimental optimisation rounds, where three distinct compositional regions (Region III ->I) with descending $I_{C}$ are clearly identified. The evolution of $I_{c}(\Theta)$ as a function of degradation time, as per Figure S3, further reveals that fast degradations of MA-rich compositions are evident after six hours of degradation tests (Region III), while two additional regions, representing compositions in local optima (Region II) and the global optima (Region I), are sequentially distinctive after 100 hours of degradation tests. Experimentally measured $I_{c}$ data reveals a $>17 \times$ reduction from the $\mathrm{MAPbI}_{3}$ end-point in Region III to the ML-optimum, at $\mathrm{Cs}_{0.17} \mathrm{MA}_{0.03} \mathrm{FA}_{0.80} \mathrm{PbI}_{3}$, in Region I. Interestingly, several MA-containing compositions show comparable $I_{C}$ to their CsFA binary cation counterparts as per Figure $3 \mathrm{~b}$. Up to $8 \%$ MA, the least chemically stable cation in the design space, can be added into the perovskite structure before environmental stability is significantly compromised.
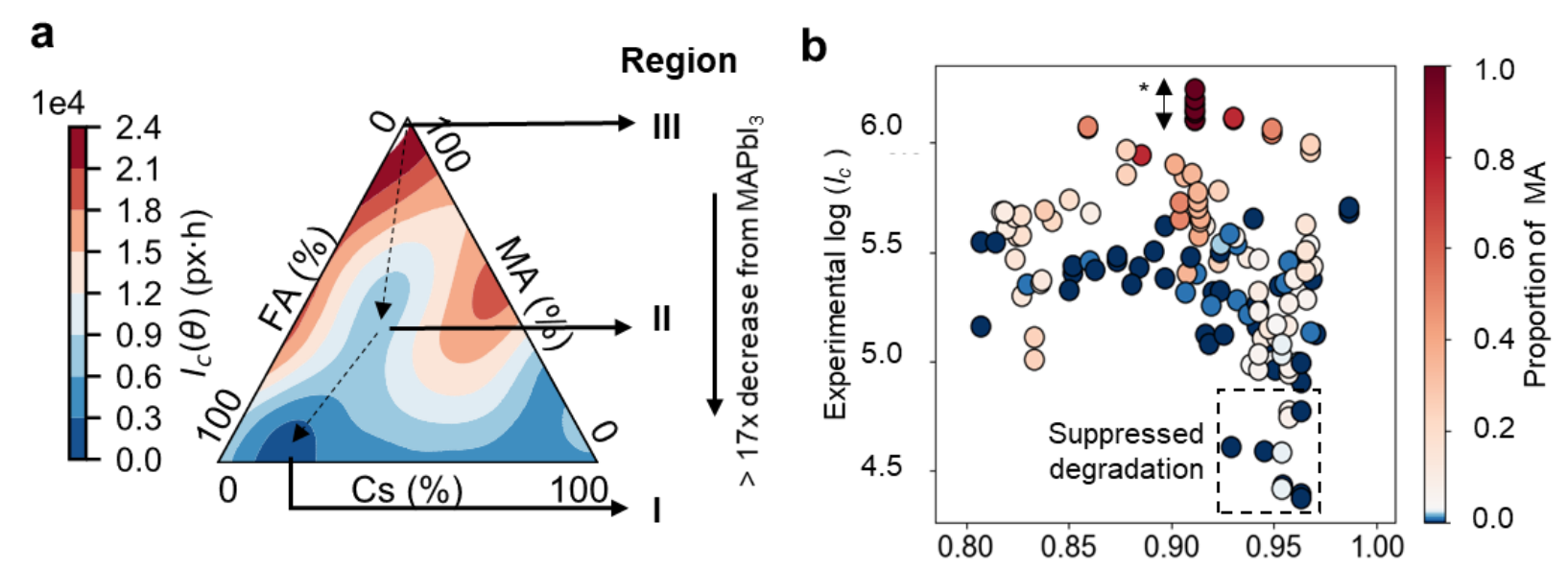

Figure 3 Composition-dependent instability landscape. a. The instability landscape $I_{c}(\Theta)$ mapped over the $\mathrm{Cs}_{\mathrm{x}} \mathrm{MA}_{\mathrm{y}} \mathrm{FA}_{1-\mathrm{x}-\mathrm{y}} \mathrm{PbI}_{3}$ compositional space evolves in time. The posterior mean of the Bayesian optimisation surrogate model estimating $I_{c}$ is shown after $6 \mathrm{~h}$ at the end of the aging test (using the experimentally measured samples from Round 0-3). Three distinct compositional regions are identified. Regions I-III are labelled following ascending order of measured $I_{C}$. b. Roles of cations in the realised degradation routes. a. $I_{C}$ of all experimentally measured samples as a function of Goldschmidt's tolerance 
factor, co-visualizing the proportion of MA. *Experimental uncertainty of measured $I_{C}$ across batches in the control composition, $\mathrm{MAPbI}_{3}$ (see Supplemental Data for full list of samples).

Figure $3 \mathrm{~b}$ visualises experimentally measured $I_{c}$ as a function of Goldschmidt's tolerance factor (TF) calculated using the average ionic radius of A-site cations in nominal compositions. TF is empirical guidance that has been widely applied to estimate the intrinsic structural stability of hybrid perovskites. ${ }^{32,33} \mathrm{We}$ find that TF optimisation is necessary but not sufficient criteria for achieving high environmental stability. During optimisation Rounds 1-3, an increasing number of compositions within a TF of $0.93-0.97$ are suggested by ML, indicating high stability of compositions with a TF of around 0.95 . This value is lower than $\mathrm{TF}=1$ of an ideal cubic structure, attributing to the incorporation of small-radius and non-volatile Cs into the $\alpha$-lattice to improve moisture and heat resistance (Figure S11).
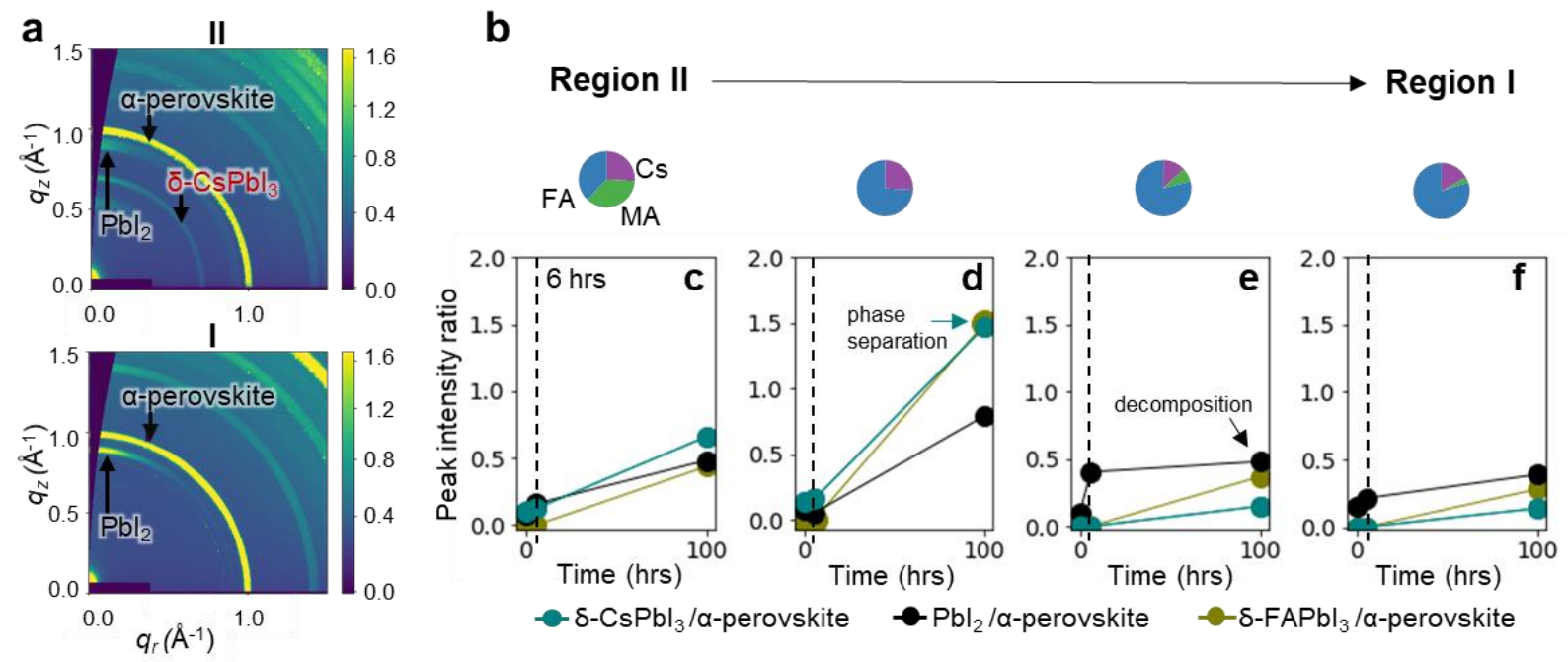

Figure 4 Mechanisms governing the divergence in degradation profiles. a. GIWAXS images of the assynthesized thin-films of $\mathrm{Cs}_{0.26} \mathrm{MA}_{0.36} \mathrm{FA}_{0.38} \mathrm{PbI}_{3}$ in Region II and the ML-optimum composition, $\mathrm{Cs}_{0.17} \mathrm{MA}_{0.03} \mathrm{FA}_{0.80} \mathrm{PbI}_{3}$, in Region I. Over-stoichiometric precursors with excess $\mathrm{PbI}_{2}$ were added in all samples following the high-efficiency perovskite solar cell recipe in ref. ${ }^{31} \mathbf{b}$. GIWAXS peak intensity ratios of the non-perovskite phases and $\mathrm{PbI}_{2}$ relative to the perovskite phase as an estimate for the extent of degradation for representative compositions in Region I and II, including c. $\mathrm{Cs}_{0.26} \mathrm{MA}_{0.36} \mathrm{FA}_{0.38} \mathrm{PbI}_{3}$ in Region II, d. $\mathrm{Cs}_{0.26} \mathrm{FA}_{0.74} \mathrm{PbI}_{3}$ at the boundary of Region I and II, e. $\mathrm{Cs}_{0.13} \mathrm{MA}_{0.8} \mathrm{FA}_{0.79} \mathrm{PbI}_{3}$, and $\mathbf{f}$. $\mathrm{Cs}_{0.17} \mathrm{MA}_{0.03} \mathrm{FA}_{0.8} \mathrm{PbI}_{3}$ in Region I. 
To validate the scientific relevance of the data fusion approach, which effectively discounts experimental sampling in the regions with high probabilities of minority phase formation, we seek to determine the impact of thermodynamics-driven minority phases on degradation dynamics. Within the $\mathrm{Cs}_{\mathrm{x}} \mathrm{MA}_{\mathrm{y}} \mathrm{FA}_{1-\mathrm{x}-\mathrm{y}} \mathrm{PbI}_{3}$, we examine the structural evolution after 0, 6- and 100-hours' degradation tests respectively using ex situ synchrotron-based grazing-incidence wide-angle scattering (GIWAXS) measurements (Figure S14-15). Comparing the ML-local optimum, (ii) $\mathrm{Cs}_{0.26} \mathrm{MA}_{0.36} \mathrm{FA}_{0.38} \mathrm{PbI}_{3}$ in Region II and the ML-optimum, and (i) $\mathrm{Cs}_{0.17} \mathrm{MA}_{0.03} \mathrm{FA}_{0.80} \mathrm{PbI}_{3}$ in Region I (Figure 4a), we observe that the two compositions (i) and (ii) exhibit comparable lattice parameters of $\alpha$-perovskites, however, local optimum (ii) contains additional $\delta$-CsPbI $\mathrm{P}_{3}$ minority phase prior to degradation tests. Interestingly, only a slightly larger increase in $\mathrm{PbI}_{2}$ is observed in (ii) than in global optimum (i) after a 6-hour degradation run. After 100 hours, (ii) exhibits a significantly bigger loss of intensity of $\alpha$-perovskites and crystallinity (Figure S14).

To understand the distinctive roles of MA, Cs, and minority phases that governs the divergence in degradation profiles behind the optimisation results, we further quantify the peak intensities of $\mathrm{PbI}_{2}$ (001), $\delta$ - $\mathrm{CsPbI}_{3}$ (002), $\mathrm{FAPbI}_{3}(001)$ and $\alpha$-perovskite (001) of four representative compositions in Region I and II (Figure 4c-f). Two composition-dependent degradation mechanisms are observed (Figure S16-17, Table S5-6). While all four samples show increased $\mathrm{PbI}_{2}$ content (evident of chemical decomposition to precursors), the emergence of $\delta$-CsPbI 3 and $\delta$ $\mathrm{FAPbI}_{3}$ minority phases in Region I films with low Cs- and MA-content (Figure 4e and f) indicates additional mechanisms of phase separation during degradation tests. We find MA plays a competing role as it accelerates chemical decomposition while suppresses phase separation (additional X-ray diffraction results shown in Figure S10). Reducing MA content from 8\% to 3\% (Figure 4e and f) show effective suppression of chemical decomposition within the first 6 hours. If we remove MA completely (Figure 4c and d), we observe a faster phase separation emerged between 6 -and 100 hours of degradation. Phase separation is observed to take place in a longer time scale compared with chemical decomposition. Overall, the stability optimisation pathway from Region II to Region I in order to achieve kinetically suppressed degradation can be summarized as follows: 1) reducing MA to suppress chemical decomposition, 2) reducing Cs to limit thermodynamics-driven minority phase formation, and 3) balancing MA, FA, and Cs for restraining additional minority phase formation in hot and humid conditions. 


\section{Insights into the Effects of Compositional Complexity on Thin-film and Device Stability}
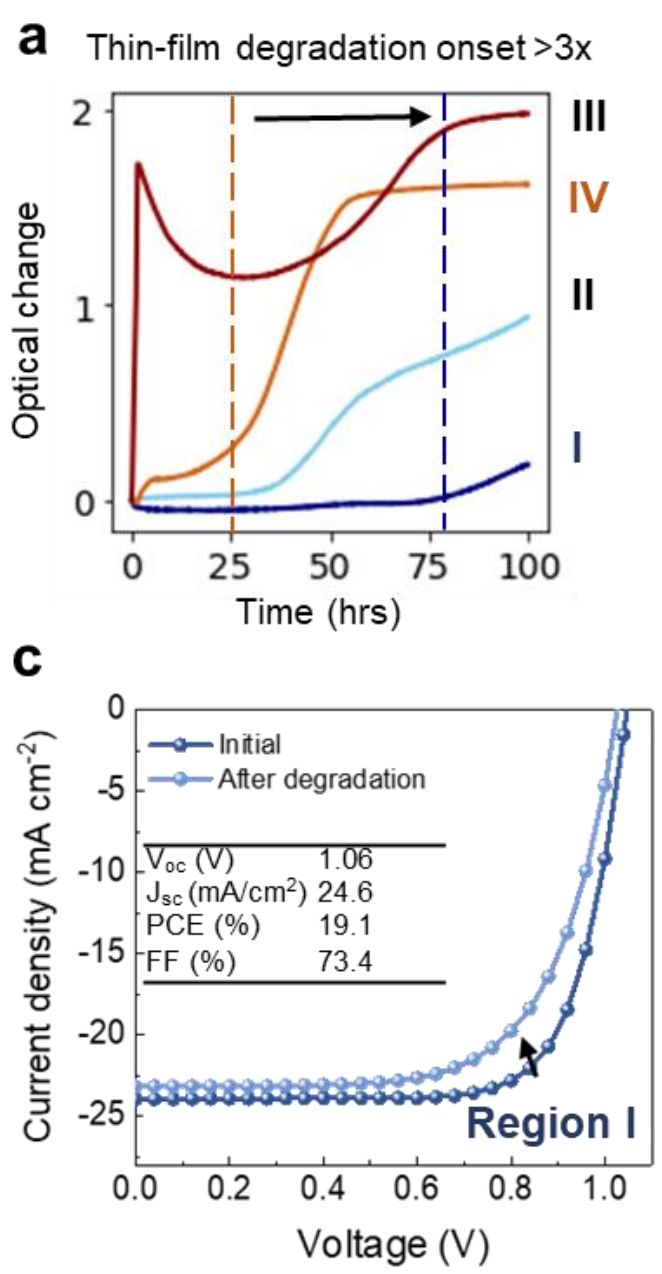

b Device degradation after damp heat test

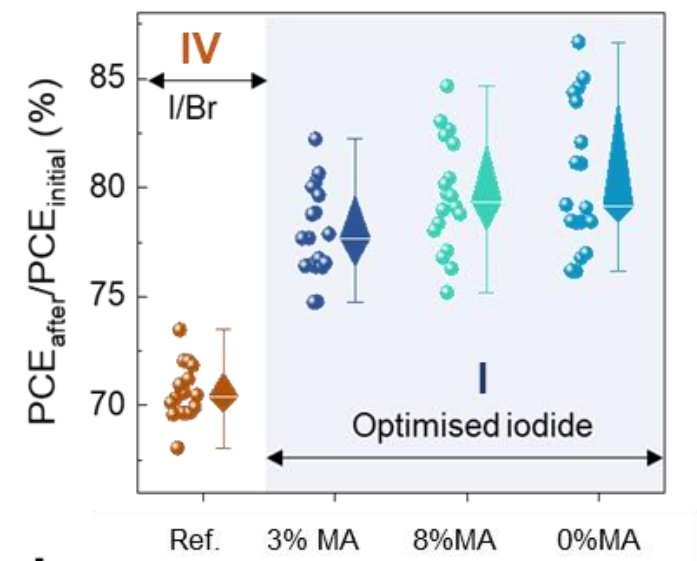

d

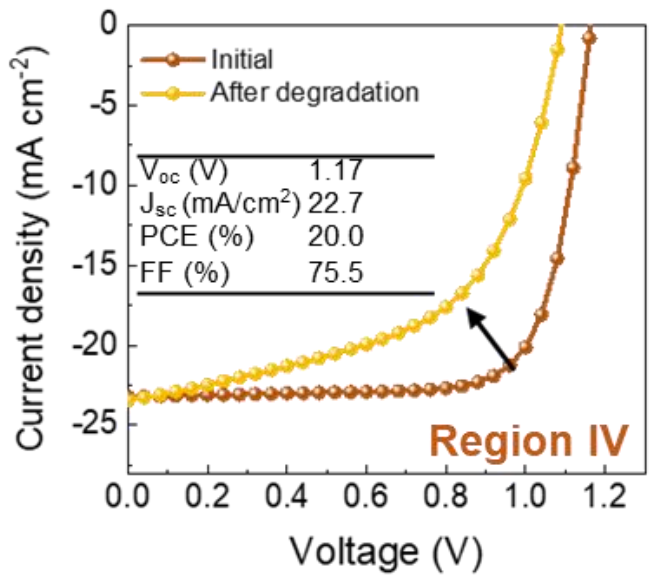

Unencapsulated under $85 \% \mathrm{RH} / 85{ }^{\circ} \mathrm{C}$ in air for 50 hours under dark

Figure 5 Suppressed degradation in thin-film and photovoltaic devices. a. Optical changes as a function of degradation time, showing the onset of degradation for representative compositions in regions I-IV, $\mathrm{Cs}_{0.17} \mathrm{MA}_{0.03} \mathrm{FA}_{0.80} \mathrm{PbI}_{3}, \mathrm{Cs}_{0.26} \mathrm{MA}_{0.36} \mathrm{FA}_{0.38} \mathrm{PbI}_{3}, \mathrm{Cs}_{0.05}\left(\mathrm{MA}_{0.17} \mathrm{FA}_{0.83}\right)_{0.95} \mathrm{~Pb}\left(\mathrm{I}_{0.83} \mathrm{Br}_{0.17}\right)_{3}(\mathrm{I} / \mathrm{Br})$, and $\mathrm{MAPbI}_{3}$ respectively. b. The percentage ratios of the solar cell efficiency after 50 hours' full damp heat degradation tests for unencapsulated devices over the initial efficiencies based on $\mathrm{I} / \mathrm{Br}$ and three ML-informed compositions in Region I, $\mathrm{Cs}_{0.17} \mathrm{MA}_{0.03} \mathrm{FA}_{0.80} \mathrm{PbI}_{3}, \mathrm{Cs}_{0.13} \mathrm{MA}_{0.08} \mathrm{FA}_{0.79} \mathrm{PbI}_{3}$, and $\mathrm{Cs}_{0.13} \mathrm{FA}_{0.87} \mathrm{PbI}_{3}$ respectively. 
c-d. Initial device efficiencies of I/Br in Region IV and the ML-optimum in Region I, and the their currentvoltage curves before and after 50 hours' accelerated degradation tests under $85 \% \mathrm{RH} / 85^{\circ} \mathrm{C}$ unencapsulated in air in dark.

To determine the impact of ML-informed compositions in the field of perovskite optoelectronics, we first demonstrate the thin-film stability improvement in this study against the state-of-the-art. Figure 5a illustrates the quantitative optical change analysis for three representative thin-film compositions from Region I, including the ML-optimum $\mathrm{Cs}_{0.17} \mathrm{MA}_{0.03} \mathrm{FA}_{0.80} \mathrm{PbI}_{3}(\mathrm{i})$, the ML-local optimum $\mathrm{Cs}_{0.26} \mathrm{MA}_{0.36} \mathrm{FA}_{0.38} \mathrm{PbI}_{3}$ (ii) and $\mathrm{MAPbI}_{3}$ (iii). We further compare the optimised fiveelement iodide perovskites (i) with a six-element iodide-bromide reference composition, $\mathrm{Cs}_{0.05}\left(\mathrm{MA}_{0.17} \mathrm{FA}_{0.83}\right)_{0.95} \mathrm{~Pb}\left(\mathrm{I}_{0.83} \mathrm{Br}_{0.17}\right)_{3}$ (iv) (I/Br). $\mathrm{I} / \mathrm{Br}$ is outside the design space of this study (referred to as Region IV), but is among the most widely employed compositions in high-efficiency perovskite solar devices. ${ }^{31,34,35}$ We found that (i) yields a $3.5 x$ lower $I_{c}$ than (iv). In addition to suppressed total degradation in optimized iodide perovskites, the degradation onset is also postponed. This is reflected by the $>3 \times$ delay in the onset of sharp optical change as per Figure 5a. The ML-optimum within the iodide perovskite space overperforms the more complex iodidebromide mix, which contradicts a long-standing assumption in the perovskite field that increasing compositional complexity entropically stabilizes the absorber. The overall environmental stability based on thin-film stability is Region I > Region II > Region IV > Region III.

We then demonstrate the enhanced stability of full photovoltaic devices without compromising conversion efficiency. To ensure rigorous environmental tests that matches the reliability requirement for perovskite solar cell commercialization, we employ air ambient, $85 \%$ relative humidity, and $85^{\circ} \mathrm{C}$ temperature full damp heat testing on non-encapsulated devices (close to the conditions of accelerated film degradation tests), which is a much harsher stress condition than most of current laboratory testing for perovskite devices. Figure $5 \mathrm{~b}$ reveals that devices of the three ML-informed compositions from Region I all overperform the state-of-the-art I/Br reference, leading to an increased efficiency (post-aging/prior-to-aging) ratio from $73 \%$ to $87 \%$ after 50 hours accelerated degradation test. While devices of both the ML-optimum and the reference composition show initial efficiencies of $>19 \%$ (as per Figure 5c and d), more severe photocurrent decay is observed in the reference. An average of the $77 \%$ of the performance is maintained in the ML-optimum in comparison to $71 \%$ in $\mathrm{I} / \mathrm{Br}$ (Figure $5 \mathrm{~b}$, over 18 devices) after degradation tests. In 
addition to the ML optimum (3\% MA), two other Region I iodide perovskites, $\mathrm{Cs}_{0.13} \mathrm{MA}_{0.08} \mathrm{FA}_{0.79} \mathrm{PbI}_{3}(8 \% \mathrm{MA})$ and $\mathrm{Cs}_{0.13} \mathrm{FA}_{0.87} \mathrm{PbI}_{3}(0 \% \mathrm{MA})$ also overperform $\mathrm{I} / \mathrm{Br}$ in both film and device stability (Figure S18). We find that compositions with fewer elements lead to improved thermo-moisture stability, where the MA-free, iodide-only composition in this comparative study achieved the highest device stability.

We should note that in a solar cell, other layers rather than the absorber within a device may also accelerate the degradation depending on the device architectures. Non-encapsulated full devices are often found to undergo faster degradations than bare films, in particular in the first several hours, attributing to interface-related degradations. ${ }^{4,19}$ In this study we only focus on the correlations between film and device stability from an angle of perovskite layer degradation without device architecture optimisation. To confirm if the device stability improvement holds under additional stress of 1Sun illumination, we further compare the photostability of devices under 1 Sun at $65^{\circ} \mathrm{C}$ in $\mathrm{N}_{2}$, (different from the conditions applied for thin-film optimisation in this study) and the results show a photo-thermostability of $8 \%$ MA > ML-optimum (3\% MA) > I/Br > 0\% MA (Figure S19). The higher device photostability of MA-containing compositions suggest a beneficial role of MA in suppressing illumination-induced degradation in multi-cation iodide perovskites.

Our device stability results highlight the non-intuitive conclusion that simplifying the perovskite formulation yields a higher device stability in hot and humid environments. This finding emphasizes the importance of achieving a holistic understanding of a compositional space to effectively identify optima. The physics-constrained sequential learning approach developed in this study can be extended to experimentally navigate higher-dimensional spaces under operational conditions, such as to identify the most environmental stable iodide-bromide-chloride perovskite alloys in the growing chemical space of $A_{\mathrm{x}} B_{\mathrm{y}} C_{z} D_{1-\mathrm{x}-\mathrm{y}-\mathrm{z}} \mathrm{Pb}\left(\mathrm{I}_{\mathrm{p}} \mathrm{Br}_{\mathrm{q}} \mathrm{Cl}_{1-\mathrm{p}-\mathrm{q}}\right)_{3}$, in order to further improve perovskite solar cells' efficiency and reliability.

\section{Conclusions}

In this study we develop a closed-loop optimisation strategy for $\mathrm{Cs}_{\mathrm{x}} \mathrm{MA}_{\mathrm{y}} \mathrm{FA}_{1-\mathrm{x}-\mathrm{y}} \mathrm{PbI}_{3}$ multi-cation perovskites against heat, moisture and light-induced degradation by introducing a physics- 
constrained Bayesian optimisation framework. We identify an FA-rich and Cs-poor region centred at $\mathrm{Cs}_{0.17} \mathrm{MA}_{0.03} \mathrm{FA}_{0.8} \mathrm{PbI}_{3}$ with $>17 \times$ stability optimisation from $\mathrm{MAPbI}_{3}$ while sampling only $1.8 \%$ of the discretized compositional space, achieving superior search efficiency and scientific relevance to brute-force screening and state-of-the-art model-free Bayesian optimisation, respectively. The study demonstrates the power of data fusion to allow material search over vast and sparsely-sampled compositional spaces, where the DFT-modelled thermodynamics serves as a probabilistic constraint and provides principled guidance to ML-directed experimentation.

We apply this physics-informed optimisation framework to achieve a holistic understanding of the fundamental composition-, phase-, and time-dependent behaviour of organic-inorganic perovskites. As a consequence of competing roles of cations in different degradation mechanisms, a composition window of up to $8 \%$ addition of the least chemically stable cation, MA, contributes to kinetically suppressed degradation, whereas the most chemically stable cation in this design space, Cs, contributes to accelerated degradation through phase separation even in the most macroscopically-stable candidates found in the $\mathrm{Cs}_{\mathrm{x}} \mathrm{MA}_{\mathrm{y}} \mathrm{FA}_{1-\mathrm{x}-\mathrm{y}} \mathrm{PbI}_{3}$ compositional space, which potentially limits the benefits of $\mathrm{Cs}$ as a perovskite stabilizing agent. These findings highlight the detrimental effects of minority phase formation, which occurs over a longer timeframe than chemical decomposition, and hence are easily underestimated in the initial compositional engineering. We further suggest several optimized iodide perovskites, including $\mathrm{Cs}_{0.17} \mathrm{MA}_{0.03} \mathrm{FA}_{0.8} \mathrm{PbI}_{3}$, and $\mathrm{Cs}_{0.13} \mathrm{MA}_{0.08} \mathrm{FA}_{0.79} \mathrm{PbI}_{3}$ that show superior photo-, thermo-, and moisture device stability to the state-of-the-art iodide-bromide mixed perovskite $\left(\mathrm{Cs}_{0.05}\left(\mathrm{MA}_{0.17} \mathrm{FA}_{0.83}\right)_{0.95} \mathrm{~Pb}\left(\mathrm{I}_{0.83} \mathrm{Br}_{0.17}\right)_{3}\right)$, providing insights into simplifying perovskite compositions for solar cell reliability.

This data fusion approach combines multiple data sources into a single search algorithm and can be utilised to include other experimental or theoretical constraints with non-negligible uncertainty into the materials design strategy. The method can be generalized to direct experimentation in other material systems, merging complementary experiments and theory to collectively inform synthesis in a closed-loop fashion across a vast chemical and structural space.

\section{Experimental Procedures}


Materials: Perovskite precursor solutions were spin-coated on UV-ozone glass substrates. Glass microscope slides (VWR) cut into square pieces were cleaned with sonication in $2 \%$ HellmanexDI water mix, DI water, and IPA respectively. We perform perovskite synthesis with overstoichiometric $\mathrm{PbI}_{2}$ in the molar ratio of $1.09\left(\mathrm{PbI}_{2}\right)$ to 1 (halide salt of CsI, MAI, and FAI). Lead (II) iodide stock solution was prepared in 9:1 N,N-dimethylformamide (Sigma-Aldrich) to dimethyl sulfoxide (Sigma-Aldrich) solvent. The perovskite precursor solution was prepared by mixing individual stock solutions following the ratios of Cs, FA, and MA suggested by the machine learning algorithm. The films were annealed at $403 \mathrm{~K}$ for 20 minutes using the central part of a hot plate in the glovebox. The spin-coating program follows a 2-step approach: $1000 \mathrm{rpm}$ for 10 seconds and acceleration of $200 \mathrm{rpm} / \mathrm{s}$, with a subsequent $6000 \mathrm{rpm}$ for 30 seconds and acceleration of $2000 \mathrm{rpm} / \mathrm{s}$. Chlorobenzene (Sigma-Aldrich) antisolvent in the quantity of $150 \mu \mathrm{L}$ was dropped 5 seconds at the beginning of the second step of spin-coating. The annealed samples were cooled to room temperature before transferring to the degradation test. $\mathrm{Cs}_{0.05}\left(\mathrm{FA}_{0.83} \mathrm{MA}_{0.17}\right)_{0.95} \mathrm{~Pb}\left(\mathrm{I}_{0.87} \mathrm{Br}_{0.13}\right)_{3}$ was synthesized following Ref. ${ }^{36}$.

Aceelerated degradation tests: Humidity, temperature, and visible light illumination level were controlled at $85 \pm 2{ }^{\circ} \mathrm{C}, 85 \pm 5 \%$, and $0.15 \pm 0.01$ Sun, respectively, using an in-house built environmental chamber. Samples were photographed automatically in every 5 min during the aging test, and additionally the humidity and temperature of the aging chamber were tracked automatically. The illumination conditions remaining stable during the aging tests was confirmed by following a printed 28-patch reference colour chart that had been placed into the picture area. After the aging test, the samples were stored in a glovebox until further characterization.

Physics-informed BO framework: In the Bayesian optimisation setting, a surrogate machine learning model, Gaussian Process (GP) regression, is used to approximate the mean and uncertainty of $I_{c}(\Theta)$ in non-sampled regions of the compositional space. Once the model is fitted, an acquisition function is used for suggesting locations in the compositional space with high chance of leading to an optimum. Our chosen base acquisition function that we modify by data fusion principle is expected improvement $\mathrm{EI}(\Theta)$,

$$
\operatorname{EI}(\Theta)=\left(\mu_{n}(\Theta)-\tau\right) \Phi\left(\frac{\mu_{n}(\Theta)-\tau}{\sigma_{n}(\Theta)}\right)+\sigma_{n}(\Theta) \phi\left(\frac{\mu_{n}(\Theta)-\tau}{\sigma_{n}(\Theta)}\right)
$$


where $\Phi$ is the standard normal cumulative distribution, $\mu_{n}(\Theta)$ is the mean of the surrogate model's (here Gaussian process regression, GP) posterior, $\tau$ is an incumbent best point, $\sigma_{n}$ is the variance of the GP model's posterior, $\phi$ is the standard normal probability distribution, and $n$ is degradation round. By maximizing $\operatorname{EI}(\Theta)$, the most promising location $\Theta^{*}$ (either due to a low expected instability index or a high uncertainty of the estimate) for the next experimental round is determined. To suggest more than the one promising composition $\Theta$ per round, we employ the local penalization algorithm to resample $\operatorname{EI}(\Theta)$, as described in ${ }^{37}$. To include the physical constraints in the Bayesian optimisation algorithm, the acquisition function is weighted according to the probabilistic model $P\left(\Delta G_{\text {mix }}(\Theta), \beta_{\text {DFT }}\right)$ following the method in ref. ${ }^{30}$ :

$$
\operatorname{EIC}(\Theta)=\operatorname{EI}(\Theta) P\left(\Delta G_{\text {mix }}(\Theta), \beta_{\mathrm{DFT}}\right)
$$

Once $\Delta \mathrm{G}_{\text {mix }}$ is modelled, the probabilistic model $P\left(\Delta G_{\text {mix }}(\Theta), \beta_{\mathrm{DFT}}\right)$ can be computed. The inherent bias and precision of DFT calculations justifies the probabilistic treatment of the constraint. Referring to the constraint value as $\Phi=P\left(\Delta G_{\text {mix }}(\Theta), \beta_{\mathrm{DFT}}\right)$, we formulate the data fusion process as maximizing the likelihood $\mathcal{L}\left(\beta_{\mathrm{DFT}} ; \Phi, \Delta G_{\mathrm{mix}}\right)$ such that:

$$
\beta_{\text {DFT }}^{*}=\operatorname{argmax}_{\beta_{\mathrm{DFT}}} \mathcal{L}\left(\beta_{\mathrm{DFT}} ; \Phi, \Delta G_{\mathrm{mix}}\right)
$$

Using a Bernoulli likelihood, this definition is equivalent to fitting a logistic regression model via maximum likelihood, with $\Delta G_{\mathrm{mix}}$ as the independent variable and the probability of phase demixing as the dependent variable. In this sense, one could estimate a certain critical energy above which the crystalline structure is unstable and will decompose into its constituent phases. In the context of convex hull stability calculations, this value is often considered to be around -0.025 eV/f.u. We hence choose $\beta_{\mathrm{DFT}}$ so that it produces cumulative probability of $P(-0.025 \mathrm{eV} /$ $\left.f . u, \beta_{\text {DFT }}^{*}\right)=0.7$ and $P\left(-0.05 e V / f . u, \beta_{\text {DFT }}^{*}\right)=0.9$. This assumption defines a smooth gradual boundary for phase mixing in the compositional space, considering the inherent uncertainty of first-principles calculations. Our choice of probabilistic model is common in machine learning literature, due to the simplicity and expressivity of logistic models. ${ }^{38}$

DFT Calculations: DFT calculations are performed within the projected augmented wave (PAW) method $^{39}$ as implemented in the VASP code. ${ }^{40}$ The Perdew Burke Ernzerhof (PBE) exchange correlation functional $^{41}$ is used with GGA and spin-orbit coupling (SOC) is included in the total 
energy calculations. Plane wave cutoff of $340 \mathrm{eV}$, and a Monkhorst-Pack k-point sampling scheme $^{42}$ is used. All degrees of freedom (cell shape, volume, and ionic positions) are relaxed in DFT calculations. Following the relaxations, A-site alloy structures at various compositions are found to retain the overall cubic symmetry. To model the phase stability of mixed A-site halide perovskites, we compute the Gibbs free energy of mixing $\left(\Delta G_{\operatorname{mix}}=\Delta H_{\text {mix }}-T \Delta S_{\text {mix }}\right)$ of these materials as a function of the A-site composition. The modeled $\Delta G_{\text {mix }}$ has two components, (1) the enthalpy of mixing $\left(\Delta H_{\mathrm{mix}}\right)$, and the entropy of mixing $\left(\Delta S_{\mathrm{mix}}\right)$. The enthalpy of mixing is calculated from DFT by taking the difference between the total energy of the mixed A-site halide perovskite with respect to the total energy of the constituent, or pure, phase. The temperature dependence $\left(T \Delta S_{\text {mix }}\right)$ to Gibbs free energy is incorporated by considering the entropic contributions associated with the configuration and rotations degrees of freedom, as discussed in detail in Ref. ${ }^{43}$. Further details of thermodynamic modeling can be found in the Ref. ${ }^{19}$

X-ray diffraction: Grazing incidence X-ray diffraction (incident angle of $1^{\circ}$ ) were performed using Rigaku SmartLab with $\mathrm{Cu}-K \alpha$ sources on the as-synthesized thin films to understand the crystal structures and to examine minority phases.

UV-visible spectroscopy:The absorptance for the films was calculated based on transmission and reflection measurements done using Perkin-Elmer Lambda 950 UV/Vis Spectrophotometer (Perkin-Elmer). Bandgaps were calculated using Tauc methods assuming direct bandgaps.

Scanning electron microscopy: The film morphology was investigated using a ZEISS Ultra-55 field-emission scanning electron microscope (FESEM, ZEISS), with InLens detector and $3.00 \mathrm{kV}$ EHT gun. The grain sizes were counted using ImageJ within the area of $\sim 0.72 \mu \mathrm{m}^{2}$. Various compositions show different distribution of grain sizes. The grain sizes of composition with high Cs $\left(\mathrm{Cs}_{0.26} \mathrm{FA}_{0.74} \mathrm{PbI}_{3}\right)$, with Cs $>20 \%$, are mostly between $300-500 \mathrm{~nm}$ and some of them reach up to $1200 \mathrm{~nm}$, indicating the presence of $\delta$-phase. The grain sizes of $\mathrm{MAPbI}_{3}$ are mostly between 200-400 nm, and reach up to $1000 \mathrm{~nm}$. The grain sizes of the rest of the compositions, which have low Cs $(<20 \%)$ are mostly between $200-400 \mathrm{~nm}$.

GIWAXS measurements: Grazing-incidence wide-angle x-ray scattering (GIWAXS) measurements were taken at beamline 11-BM (CMS) at the National Synchrotron Light Source II (NSLS-II) of Brookhaven National Laboratory. The x-ray beam with the energy of $13.5 \mathrm{keV}$ shone 
on the perovskite films in the grazing incident geometry. The data presented in the study was taken at incident angle $\theta=0.2$ which probes the bulk structure of the films. The scattering spectra were collected with the exposure time of 30 seconds by an area detector (DECTRIS Pilatus 800K) placed $257 \mathrm{~mm}$ away from the sample. The data analysis was performed by using custom-made software SciAnalysis. ${ }^{44}$

Device fabrication: Unless stated otherwise, all materials were purchased from Sigma Aldrich or Merck and used as received. MAI and FAI were purchased from $\mathrm{Xi}^{\prime}$ an P-OLED. $\mathrm{PbI}_{2}$ was purchased from Lumtec. The Ta- $\mathrm{WO}_{\mathrm{x}}$ colloidal solution was purchased from Avantama Ltd. The $\mathrm{SnO}_{2}$-PEIE solution was prepared by mixing $15 \mathrm{wt} \% \mathrm{SnO}_{2}$ aqueous solution $(300 \mu \mathrm{L})$ with 1.8 $\mathrm{mL}$ of isopropanol and $\mathrm{H}_{2} \mathrm{O}(1 / 1, \mathrm{v} / \mathrm{v})$ and $20 \mu \mathrm{L}$ of PEIE. First, ITO substrates were sonicated in acetone/isopropanol for 10 minutes/5 minutes, respectively. Before spin coating $\mathrm{SnO}_{2}$-PEIE solution $(80 \mu \mathrm{L})$ at $3500 \mathrm{rpm}$ for 30 seconds, the ITO substrates were treated by UV-Ozone for 10 minutes in ambient air. After annealling at $150^{\circ} \mathrm{C} / 10$ minutes in ambient air, $80 \mu \mathrm{L}$ of PCBM:PMMA solution was spin-coated on a $\mathrm{SnO}_{2} / \mathrm{PEIE}$ layer at $2000 \mathrm{rpm}$ for 30 seconds and then annealed at $150^{\circ} \mathrm{C}$ for 10 minute. $1.2 \mathrm{M} \mathrm{PbI}_{2}$ and FAI solution was prepared first in DMF and DMSO (4:1 v/v), and 1.2 M MAI/CsI solution was prepared in DMSO. The $\mathrm{MA}_{x} \mathrm{Cs}_{y} \mathrm{FA}_{1-x-y} \mathrm{PbI}_{3}$ precursors were prepared by mixing the mother solutions in the target ratio. The perovskite precursor soloution was spin-coated on the PCBM substrate using the following parameters: 200 rpm for 2 seconds, $2000 \mathrm{rpm}$ for 2 seconds, and $5000 \mathrm{rpm}$ for 40 seconds ( $\mathrm{a}=3$ seconds). Then, $180 \mu \mathrm{L}$ of chlorobenzene was dropped on the film at 20 seconds, followed by annealing at $110^{\circ} \mathrm{C}$ for 10 minutes and $150^{\circ} \mathrm{C}$ for 5 minutes. PDCBT as a hole transporting layer was spin-coated at $2000 \mathrm{rpm}$ for 40 seconds and annealed at $90^{\circ} \mathrm{C}$ for 5 minutes. Finally, $100 \mu \mathrm{L}$ of Ta-WOx was coated on PDCBT at $2000 \mathrm{rpm}$ for 30 seconds and annealed at $75^{\circ} \mathrm{C}$ in ambient air. A 100-nmthick Au electrode was deposited through a shadow mask via thermal evaporation. For the devices used in the stability tests, a $200 \mathrm{~nm}$ Au layer was deposited.

Device characterization: The $J-V$ curves of the solar cells were obtained using a Keithley source under $100 \mathrm{~mW} \mathrm{~cm}^{-2} \mathrm{AM} 1.5 \mathrm{G}$ illumination. The $J-V$ characteristics were measured from -0.1 to 1.2 $\mathrm{V}$ (forward scan) at a scan rate of $20 \mathrm{mV} / \mathrm{s}$. No hysteresis is observed in the devices. For the thermal stability test at $85^{\circ} \mathrm{C} / 85 \% \mathrm{RH}$, the devices were stored in a sample box in a climate chamber in the dark without any encapsulation. The devices were tested before and after storing in the climate 
chamber for 50 hours. For the photo-stability test at $85^{\circ} \mathrm{C}$, the devices were stored in a sealed chamber with $\mathrm{N}_{2}$ flow under metal halide light illumination $\left(100 \mathrm{~mW} \mathrm{~cm}^{-2}\right)$. The devices were continueously measured with forward scan at a scan rate of $20 \mathrm{mV} / \mathrm{s}$.

\section{Data and Code Availability}

Details of materials, experimental methods, machine learning framework and camera-based in situ degradation monitoring platform details are available in Supplemental information. The codes and the datasets used for Bayesian optimisation are available in GitHub repository https:/github.com/PV-Lab/SPProC. Lists of samples and raw data of XRD characterization, and thin-film degradation results are shown in Supplemental Data.

\section{Acknowledgments}

The authors thank members of MIT Photovoltaics Research Laboratory, in particularly Jim Serdy for setting up and maintaining the environmental chamber for accelerated aging tests; Antonio Buscemi and Isaac Metcalf for assistance in XRD measurements, Isaac Siyu Tian for initial image processing, and Shreyaa Raghavan for the user-friendly Python package development. SS thanks Dr. Masafumi Fukuto and Dr. Kevin Yager from Brookhaven National Laboratory for beamtime assistance. High-throughput GIWAXS images were collected at BM-11, NSLS-II. The authors thank Prof. Joshua Schrier (Fordham university) for the discussion on perovskite material discovery using machine learning techniques. SS further thanks Felice Frankel for valuable insights on graphic visualization, and Mariela Castillo for early-stage project management.

This study is based upon work supported by the Defense Advanced Research Projects Agency (DARPA) under Contract No. HR001118C0036. Any opinions, findings and conclusions or recommendations expressed in this material are those of the authors and do not necessarily reflect the views of DARPA. SS, NTPH, TB thank TOTAL S.A., US National Science Foundation grant CBET-1605547, and the Skoltech NGP program. AT was supported by Alfred Kordelin Foundation and Svenska Tekniska Vetenskaps-akademien i Finland. FO was supported by the U.S. 
Department of Energy under Photovoltaic Research and Development program under Award DEEE0007535. This research used computational resources sponsored by the DOE Office of Energy Efficiency and Renewable Energy and located at National Renewable Energy Laboratory. YZ, TH and CJB gratefully acknowledges the financial support through the "Aufbruch Bayern" initiative of the state of Bavaria (EnCN and "Solar Factory of the Future"), the Bavarian Initiative "Solar Technologies go Hybrid” (SolTech), "ELF-PV Design and development of solution processed functional materials for the next generations of PV technologies" by the Bavarian State Government (No. 44-6521a/20/4) and the DFG, Projects No. 182849149 - SFB 953, IGK2495 and INST 90/1093/1. JJY and MB were supported by the Institute for Soldier Nanotechnology (ISN) grant W911NF-13-D-0001, the National Aeronautics and Space Administration (NASA) grant NNX16AM70H, and the Eni-MIT Alliance Solar Frontiers Center.

\section{Author Contributions}

SS and TB conceived the project. SS designed the experiments and led the laboratory and synchrotron-based measurements. AT developed colour and chamber environment calibration. AT and LZ processed the camera data. FO and JF designed the Bayesian optimisation algorithms and instability index, and FO, AT, and LZ wrote the Bayesian optimisation implementation. JT synthesized all thin-films samples supervised by SS. YZ fabricated the devices. JT and NTPH performed laboratory structural and morphological characterisation. JT, AT, NTPH performed the film degradation tests. AG performed DFT calculations. TH, YZ and IMP performed device degradation tests. FO, AT, LZ, and JF developed the incorporation of DFT into the Bayesian optimisation loop, and AT implemented the Gaussian process model and probability distribution on phase stability. AE built the automatic humidity control supervised by SS and AT. CB and AT developed the camera control program and visualized degradation data. CB performed optical characterisation supervised by SS. SS, JT, NTPH, and JJY performed structural characterisation using synchrotron radiation. RL assisted the measurements at beamline BM-11, NSLS-II. ZR performed the teacher-student model for DFT and non-DFT optimisation comparison. CJB, MB, VS, JF and TB supervised the project. SS, AT, FO, and TB wrote the manuscript with contributions from all co-authors. All authors discussed the results and inferred the implications of the project. 


\section{Declaration of Interests}

Authors declare no competing interests.

\section{References and Notes}

1. Boyd, C.C., Cheacharoen, R., Leijtens, T., and McGehee, M.D. (2019). Understanding Degradation Mechanisms and Improving Stability of Perovskite Photovoltaics. Chem. Rev. 119, 3418-3451.

2. Jeon, N.J., Noh, J.H., Yang, W.S., Kim, Y.C., Ryu, S., Seo, J., and Seok, S. Il (2015). Compositional engineering of perovskite materials for high-performance solar cells. Nature 517, 476-480.

3. Saliba, M. (2019). Polyelemental, Multicomponent Perovskite Semiconductor Libraries through Combinatorial Screening. Adv. Energy Mater. 9, 1803754.

4. Khenkin, M. V., Katz, E.A., Abate, A., Bardizza, G., Berry, J.J., Brabec, C., Brunetti, F., Bulović, V., Burlingame, Q., Di Carlo, A., et al. (2020). Consensus statement for stability assessment and reporting for perovskite photovoltaics based on ISOS procedures. Nat. Energy 5, 35-49.

5. Rohr, B., Stein, H.S., Guevarra, D., Wang, Y., Haber, J.A., Aykol, M., Suram, S.K., and Gregoire, J.M. (2020). Benchmarking the acceleration of materials discovery by sequential learning. Chem. Sci. 11, 2696-2706.

6. Zhang, W., Mao, J., Li, S., Chen, Z., and Guo, Z. (2017). Phosphorus-Based Alloy Materials for Advanced Potassium-Ion Battery Anode. J. Am. Chem. Soc. 139, 33163319.

7. George, E.P., Raabe, D., and Ritchie, R.O. (2019). High-entropy alloys. Nat. Rev. Mater. 4, 515-534.

8. Zhong, M., Tran, K., Min, Y., Wang, C., Wang, Z., Dinh, C.T., De Luna, P., Yu, Z., Rasouli, A.S., Brodersen, P., et al. (2020). Accelerated discovery of CO2 electrocatalysts using active machine learning. Nature 581, 178-183.

9. Kumar, G., Bossert, H., McDonald, D., Chatzidimitriou, A., Ardagh, M.A., Pang, Y., Lee, C.S., Tsapatsis, M., Abdelrahman, O.A., and Dauenhauer, P.J. (2020). Catalysis-in-a-Box: Robotic Screening of Catalytic Materials in the Time of COVID-19 and Beyond. Matter 3, 805-823.

10. Burger, B., Maffettone, P.M., Gusev, V. V., Aitchison, C.M., Bai, Y., Wang, X., Li, X., Alston, B.M., Li, B., Clowes, R., et al. (2020). A mobile robotic chemist. Nature 583, 237-241. 
11. Cole, J.M. (2020). A Design-to-Device Pipeline for Data-Driven Materials Discovery. Acc. Chem. Res. 53, 599-610.

12. Gómez-Bombarelli, R., Aguilera-Iparraguirre, J., Hirzel, T.D., Duvenaud, D., Maclaurin, D., Blood-Forsythe, M.A., Chae, H.S., Einzinger, M., Ha, D.G., Wu, T., et al. (2016). Design of efficient molecular organic light-emitting diodes by a high-throughput virtual screening and experimental approach. Nat. Mater. 15, 1120-1127.

13. Kirman, J., Johnston, A., Kuntz, D.A., Askerka, M., Gao, Y., Todorović, P., Ma, D., Privé, G.G., and Sargent, E.H. (2020). Machine-Learning-Accelerated Perovskite Crystallization. Matter 2, 938-947.

14. Li, Z., Najeeb, M.A., Alves, L., Sherman, A., Parrilla, P.C., Pendleton, I.M., Zeller, M., Schrier, J., Norquist, A.J., and Chan, E. (2019). Robot-Accelerated Perovskite Investigation and Discovery (RAPID): 1. Inverse Temperature Crystallization.

15. MacLeod, B.P., Parlane, F.G.L., Morrissey, T.D., Häse, F., Roch, L.M., Dettelbach, K.E., Moreira, R., Yunker, L.P.E., Rooney, M.B., Deeth, J.R., et al. (2020). Self-driving laboratory for accelerated discovery of thin-film materials. Sci. Adv. 6, eaaz8867.

16. Xue, D., Balachandran, P. V., Yuan, R., Hu, T., Qian, X., Dougherty, E.R., and Lookman, T. (2016). Accelerated search for BaTiO3-based piezoelectrics with vertical morphotropic phase boundary using Bayesian learning. Proc. Natl. Acad. Sci. U. S. A. 113, 1330113306.

17. Curtarolo, S., Hart, G.L.W., Nardelli, M.B., Mingo, N., Sanvito, S., and Levy, O. (2013). The High-throughput Highway to Computational Materials Design. Nat. Mater. 12, 191201.

18. Jesper Jacobsson, T., Correa-Baena, J.-P., Pazoki, M., Saliba, M., Schenk, K., Grätzel, M., and Hagfeldt, A. (2016). Exploration of the compositional space for mixed lead halogen perovskites for high efficiency solar cells. Energy Environ. Sci. 9, 1706-1724.

19. Schelhas, L.T., Li, Z., Christians, J.A., Goyal, A., Kairys, P., Harvey, S.P., Kim, D.H., Stone, K.H., Luther, J.M., Zhu, K., et al. (2019). Insights into operational stability and processing of halide perovskite active layers. Energy Environ. Sci. 12, 1341-1348.

20. SheffieldML/GPyOpt: Gaussian Process Optimization using GPy https://github.com/SheffieldML/GPyOpt.

21. Zhu, X.H., Wei, Z.R., Jin, Y.R., and Xiang, A.P. (2007). Growth and characterization of a PbI2 single crystal used for gamma ray detectors. Cryst. Res. Technol. 42, 456-459.

22. Hu, Y., Bai, F., Liu, X., Ji, Q., Miao, X., Qiu, T., and Zhang, S. (2017). Bismuth Incorporation Stabilized $\alpha-\mathrm{CsPbI} 3$ for Fully Inorganic Perovskite Solar Cells. ACS Energy Lett. 2, 2219-2227.

23. Masi, S., Echeverría-Arrondo, C., Salim, K.M.M., Ngo, T.T., Mendez, P.F., LópezFraguas, E., Macias-Pinilla, D.F., Planelles, J., Climente, J.I., and Mora-Seró, I. (2020). Chemi-Structural Stabilization of Formamidinium Lead Iodide Perovskite by Using Embedded Quantum Dots. ACS Energy Lett., 418-427. 
24. Hashmi, S.G., Tiihonen, A., Martineau, D., Ozkan, M., Vivo, P., Kaunisto, K., Ulla, V., Zakeeruddin, S.M., and Grätzel, M. (2017). Long term stability of air processed inkjet infiltrated carbon-based printed perovskite solar cells under intense ultra-violet light soaking. J. Mater. Chem. A 5, 4797-4802.

25. Hashmi, S.G., Martineau, D., Li, X., Ozkan, M., Tiihonen, A., Dar, M.I., Sarikka, T., Zakeeruddin, S.M., Paltakari, J., Lund, P.D., et al. (2017). Air Processed Inkjet Infiltrated Carbon Based Printed Perovskite Solar Cells with High Stability and Reproducibility. Adv. Mater. Technol. 2, 1600183.

26. Stoddard, R.J., Dunlap-Shohl, W.A., Qiao, H., Meng, Y., Kau, W.F., and Hillhouse, H.W. (2020). Forecasting the Decay of Hybrid Perovskite Performance Using Optical Transmittance or Reflected Dark-Field Imaging. ACS Energy Lett. 5, 946-954.

27. Kim, S., Eom, T., Ha, Y.-S., Hong, K.-H., and Kim, H. (2020). Thermodynamics of Multicomponent Perovskites: A Guide to Highly Efficient and Stable Solar Cell Materials. Chem. Mater. 32, 4265-4272.

28. Herbol, H.C., Poloczek, M., and Clancy, P. (2020). Cost-effective materials discovery: Bayesian optimization across multiple information sources. Mater. Horizons.

29. Doan, H.A., Agarwal, G., Qian, H., Counihan, M.J., Rodríguez-López, J., Moore, J.S., and Assary, R.S. (2020). Quantum Chemistry-Informed Active Learning to Accelerate the Design and Discovery of Sustainable Energy Storage Materials. Chem. Mater.

30. Gelbart, M.A., Snoek, J., and Adams, R.P. (2014). Bayesian optimization with unknown constraints. In Uncertainty in Artificial Intelligence - Proceedings of the 30th Conference, UAI 2014, pp. 250-259.

31. Saliba, M., Matsui, T., Seo, J.-Y., Domanski, K., Correa-Baena, J.-P., Nazeeruddin, M.K., Zakeeruddin, S.M., Tress, W., Abate, A., Hagfeldt, A., et al. (2016). Cesium-containing triple cation perovskite solar cells: improved stability, reproducibility and high efficiency. Energy Environ. Sci. 9, 1989-1997.

32. Kieslich, G., Sun, S., and Cheetham, A.K. (2014). Solid-state principles applied to organic-inorganic perovskites: new tricks for an old dog. Chem. Sci. 5, 4712-4715.

33. Li, Z., Yang, M., Park, J.-S., Wei, S.-H., Berry, J.J., and Zhu, K. (2016). Stabilizing Perovskite Structures by Tuning Tolerance Factor: Formation of Formamidinium and Cesium Lead Iodide Solid-State Alloys. Chem. Mater. 28, 284-292.

34. Correa-Baena, J.P., Saliba, M., Buonassisi, T., Grätzel, M., Abate, A., Tress, W., and Hagfeldt, A. (2017). Promises and challenges of perovskite solar cells. Science 358, 739_744.

35. Saidaminov, M.I., Williams, K., Wei, M., Johnston, A., Quintero-Bermudez, R., Vafaie, M., Pina, J.M., Proppe, A.H., Hou, Y., Walters, G., et al. (2020). Multi-cation perovskites prevent carrier reflection from grain surfaces. Nat. Mater. 19, 412-418.

36. Correa-Baena, J.-P., Luo, Y., Brenner, T.M., Snaider, J., Sun, S., Li, X., Jensen, M.A., Hartono, N.T.P., Nienhaus, L., Wieghold, S., et al. (2019). Homogenized Halides and 
Alkali Cation Segregation in Alloyed Organic-inorganic Perovskites. Science 363, 627631.

37. González, J., Dai, Z., Hennig, P., and Lawrence, N. Batch Bayesian Optimization via Local Penalization.

38. Goodfellow, I., Bengio, Y., and Courville, A. (2016). Deep Learning (MIT press Cambridge).

39. Blöchl, P.E. (1994). Projector augmented-wave method. Phys. Rev. B 50, 17953-17979.

40. Kresse, G., and Furthmüller, J. (1996). Efficiency of ab-initio total energy calculations for metals and semiconductors using a plane-wave basis set. Comput. Mater. Sci. 6, 15-50.

41. Perdew, J.P., Burke, K., and Ernzerhof, M. (1996). Generalized Gradient Approximation Made Simple. Phys. Rev. Lett. 77, 3865-3868.

42. Monkhorst, H.J., and Pack, J.D. (1976). Special points for Brillouin-zon integrations. Phys. Rev. B 13, 5188-5192.

43. Blöchl, P.E. (1994). Projector augmented-wave method. Phys. Rev. B 50, 17953-17979.

44. https://github.com/CFN-softbio/SciAnalysis. 


\section{Supplemental Information}

\section{Supplemental Methods}

\section{Thin-film Deposition}

In total, 202 perovskite thin-films were synthesised, and 196 of which were degraded in this study over 7 degradation rounds, including one repeated round, as shown in the main text. Of the 202 films synthesized across all rounds, 99 films were distinctly unique in composition, 95 of which were characterized by laboratory grazing incidence X-ray diffraction with 7 compositions were further characterized after 6 and 100 hours of degradation tests, respectively, using grazing-incidence wide-angle scattering. Amongst the final selected seven compositions for advanced characterization, the bromine-containing composition, $\mathrm{Cs}_{0.05}\left(\mathrm{FA}_{0.83} \mathrm{MA}_{0.17}\right)_{0.95} \mathrm{~Pb}\left(\mathrm{I}_{0.87} \mathrm{Br}_{0.13}\right)_{3}$, was synthesized following a distinct recipe from Ref ${ }^{1}$. The halide perovskite precursor solutions for the composition were prepared by mixing FAI and CsI salts with $\mathrm{PbI}_{2}$ solution, separately, and MAI with $\mathrm{PbBr}_{2}$ solution, using the same method and molarity ratios as described above. All prepared precursors solutions were mixed together in the volume ratios listed for FA, MA and Cs. Full list of samples and synthetic parameters are shown in Supplementary Data.

\section{Thin-film Degradation}

In total 196 individual thin-films within 7 experimental rounds were degraded in this study, including one initial round, three optimization rounds, and three rounds for validation and advanced characterization. The sample holder was made of graphite for maximum heat transfer, painted with medium grey colour for preventing over- or under saturating of the pictures taken, and designed to minimize reflections from the samples to the camera. Sample holder was heated from the bottom and its temperature was set to $85^{\circ} \mathrm{C}$ with SOLO 4824 temperature controller. The temperature was controlled using a thermocouple wired into the sample holder. The chamber was illuminated using Advanced Illumination DL097 LED lamp and Advanced Illumination ICS 2.0 LED controller. The lamp provided even visible light only illumination. In this study, the illumination acts foremost as a necessary lighting of the samples for the camera, and also as a degradation factor. Air humidity in the chamber was controlled and monitored using a control program running on Arduino, Si7021 humidity sensor, and water evaporation unit utilizing a fan. The stability of the humidity sensor during the aging tests was followed and the chamber conditions were tracked using EasyLog EL-USB-2 humidity-temperature logger. Both humidity sensor and logger were located close to the samples but with distance of centimetres from any surface possibly condensing water. 
In situ Optical Imaging

Samples were photographed using ThorLabs DCC1645C camera (with a removed IR filter to maximize data collection bandwidth of the camera) and ThorLabs MVL6WA lens, providing a 3-band approximation of the colour of the sample. The photographs of the samples were taken in BMP format in every 5 minutes using a control program implemented using LabVIEW. The photographs were saved automatically directly to Dropbox folder for further analysis. The photographs of the samples were automatically sliced into pictures of individual samples and colour calibrated to ensure reproducible and repeatable collection of quantitative colour data. The data was used for determining the instability index for each sample as shown in the main article. The variations in the Instability Index $\left(I_{c}\right)$ values arise from several sources: the variations between the sample replicates, variations in the environmental conditions between the sample holder locations, and in how camera sees the colour of the samples (e.g., reflections decrease the accuracy of colour determination). Experimental uncertainty is highly dependent on the composition and varies between $5000 \mathrm{px} * \min$ and $225000 \mathrm{px} * \min$. The estimated error for our optimum region (Region I) is 9000 px*min (with $I_{c}$ of approximately $30000 \mathrm{px} * \min$ ). On average 7284 pixels were captured per thin film samples of $2.54 \mathrm{~cm} \times 1.27 \mathrm{~cm}$, leading to an estimated resolution of $210 \mu \mathrm{m}$.

Colour calibration by transforming the sample colours to a stable reference colour space ensures the colours are comparable even when the pictures are taken under different illumination conditions or with different camera-lens setups. This was done utilizing an X-Rite Colour Checker Passport with 28 reference colour patches that was photographed at the beginning of each aging test in the aging chamber. During the aging tests, camera settings were defined to be as illuminated as possible without oversaturating the white colour patch in Xrite colour chart, ensuring that the colour calibration procedure succeeds without distortions. The sample colours were transformed into a larger $\mathrm{L}^{*} \mathrm{a}^{*} \mathrm{~b}$ colour space and colour warped by 3D thin-plate spline that has been shown to be among the most accurate colour warping methods for colour calibration. ${ }^{2}$ Distortion between the colours of the reference colour chart in real and reference colour spaces is defined as:

$$
D=\frac{\left[\begin{array}{c}
V \\
O(4,3)
\end{array}\right]}{\left[\begin{array}{cc}
K & P \\
P^{T} & O(4,4)
\end{array}\right]}
$$

where $O(n, m)$ is a zero matrix with shape $m \times n, V$ represents the colours of the reference colour chart in the reference space (obtained from the colour chart manufacturer), matrix $P$ represents the colours of the reference colour chart in the original space, and matrix $K$ is the distortion between the colour patches in the reference and real colour spaces. The definitions for these matrices are: 


$$
\begin{aligned}
V & =\left[\begin{array}{cccc}
1 & x_{1}^{\prime} & y_{1}^{\prime} & z_{1}^{\prime} \\
\vdots & \vdots & \vdots & \vdots \\
1 & x_{N}^{\prime} & y_{N}^{\prime} & z_{N}^{\prime}
\end{array}\right], \\
P & =\left[\begin{array}{cccc}
1 & x_{1} & y_{1} & z_{1} \\
\vdots & \vdots & \vdots & \vdots \\
1 & x_{N} & y_{N} & z_{N}
\end{array}\right], \\
K & =\left[\begin{array}{ccc}
0 & \ldots & U\left(r_{1 N}\right) \\
U\left(r_{21}\right) & \ldots & U\left(r_{2 N}\right) \\
\vdots & \vdots & \vdots \\
U\left(r_{N 1}\right) & \ldots & 0
\end{array}\right],
\end{aligned}
$$

where $N$ is the number of colour patches (in our reference colour chart $N=24$ ) and $U$ is defined as:

$$
U\left(r_{i j}\right)=2 r_{i j}^{2} \log \left(r_{i j}+10^{-20}\right)
$$

(with a constant value added for numerical stability) and the Euclidian distance between the two colours is:

$$
r_{i j}=\sqrt{\left(x_{i}-x_{j}\right)^{2}+\left(y_{i}-y_{j}\right)^{2}+\left(z_{i}-z_{j}\right)^{2}}
$$

where $x_{i}, y_{i}, z_{i}$ and $x_{j}, y_{j}, z_{j}$ are the three colour components of each colour (in this study the components are $L, a$, and $b$ in Lab colour space) in the real colour space and reference colour space, respectively.

Applying the same distortion $D$ to the samples completes the colour warping:

$$
\left[\begin{array}{c}
V_{s} \\
O(4,3)
\end{array}\right]=\left[\begin{array}{cc}
K_{s} & P_{s} \\
P_{S}^{T} & O(4,4)
\end{array}\right] D
$$

where matrices $V_{\mathrm{s}}$ and $P_{\mathrm{s}}$ represent the colours of the samples in the reference and original space, respectively, and matrix $K_{\mathrm{s}}$ represents the distortion between the colours of the samples and reference colour chart. The shape distortion between the colours of the samples and the reference colour chart patches in the real space is:

$$
K_{S}=\left[\begin{array}{ccc}
0 & \ldots & U\left(r_{s_{1}, c_{N}}\right) \\
U\left(r_{s_{2}, c_{1}}\right) & \ldots & U\left(r_{s_{2}, c_{N}}\right) \\
\vdots & \vdots & \vdots \\
U\left(r_{s_{M}, c_{1}}\right) & \ldots & 0
\end{array}\right],
$$

where $s_{i}$ refer to sample colours, $M$ is the number of samples, and $c_{i}$ refer to reference colour chart colours. Matrices $V_{S}$ and $P_{S}$ that represent colours of the samples in the reference and real colour spaces, respectively, are:

$$
V=\left[\begin{array}{cccc}
1 & x_{1}^{\prime} & y_{1}^{\prime} & z_{1}^{\prime} \\
\vdots & \vdots & \vdots & \vdots \\
1 & x_{M}^{\prime} & y_{M}^{\prime} & z_{M}^{\prime}
\end{array}\right],
$$




$$
P=\left[\begin{array}{cccc}
1 & x_{1} & y_{1} & z_{1} \\
\vdots & \vdots & \vdots & \vdots \\
1 & x_{M} & y_{M} & z_{M}
\end{array}\right] .
$$

After colour calibration, the sample colours were transformed back to RGB space.

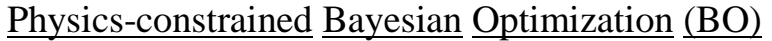

Bayesian optimization is used to find $\Theta^{*}$, the perovskite composition with smallest instability index $I_{c}$, using as few samples as possible, according to:

$$
\Theta^{*}=\operatorname{argmin}_{\Theta} \mathbb{E}\left[I_{\mathrm{c}}(\Theta)\right] \text { s.t. } P\left(\Delta G_{\text {mix }}(\Theta), \beta_{\mathrm{DFT}}\right)>0
$$

where $\Theta^{*}=\operatorname{argmin}_{\Theta} \mathrm{E}\left[I_{\mathrm{c}}(\Theta)\right]$ is a $\mathrm{BO}$ framework optimising the $I_{\mathrm{c}}$, and $I_{\mathrm{c}}(\Theta)$ is a noisy, black-box function that we evaluate by making samples at different $\Theta$ compositions. We propose to use the probabilistic constraints from DFT-computations given by $P\left(\Delta G_{\text {mix }}(\Theta), \beta_{\text {DFT }}\right)$. The probabilistic constraint models the probability of phase mixing according to Gibbs free energy $\Delta G_{\text {mix }}(\Theta)$ and data-fusion factor $\beta_{\mathrm{DFT}}$.

$\underline{\text { Gibbs Free Energy of Mixing Calculations }}$ (DFT)

Alloy structures are created using the pseudo-cubic as the starting structure for the pure compositions (obtained from Ref. ${ }^{3}$ ) with random substitution at the A-site. Special quasi-random structure (SQS) method, ${ }^{4}$ as implemented in the ATAT package, ${ }^{5,6}$, is used to obtain structures for various A-site alloy compositions. Two different supercell sizes 96 and 144-atoms are used and for each A-site composition with multiple (two or three) structures, varying in the orientation of the MA and FA molecules, are considered. As benchmarked in Ref. ${ }^{7}$, the reproducibility and precision in our DFT total energy calculations is very high. The variability in the computed value of Gibbs free energy at a specific composition is between $5-20 \mathrm{meV} / \mathrm{unit}$, and it comes from the varying orientation of the FA and MA molecules between the multiple structures, considered in our simulations.

\section{$\underline{\text { Regression Model for DFT Calculations }}$}

Calculating $\Delta G_{\text {mix }}$ for ternary compounds is computationally expensive and infeasible over the whole composition space; therefore, $\Delta G_{\text {mix }}$ is modelled using an auxiliary GP regression model trained on 91 DFTcomputed $\Delta G_{\text {mix }}$ from 47 binary perovskite compositions. Based on the DFT data, the $\Delta G_{\text {mix }}(\Theta)$ is approximated over the ternary composition space (modelled values for the end members shown in Figure S6). The auxiliary GP model uses a radial basis function (RBF) kernel with initialized length scale of 
0.003 (eV/f.u. ) and variance of 0.025 (eV/f. u. $)^{2}$. The kernel, initial length scale and variance values were determined based on grid search, and optimized according to maximum likelihood. The model was validated using leave-one-out cross validation, which resulted in the mean squared error MSE $=$ 0.0004 (eV/f. u. $)^{2}$. Additionally, the final model was tested against three datapoints that are out of the validation dataset (ternary triple cation phase stability calculations in Ref. $^{8}$ ), resulting in MSE = 0.00015 (eV/f. u. $)^{2}$. Both MSE values are low in comparison to the $\Delta \mathrm{G}_{\mathrm{mix}}$ of the training set, which shows that the model regresses $\Delta \mathrm{G}_{\text {mix }}$ successfully for ternary compositions. The key sources of uncertainty in this study relating to incorporating DFT calculations into the experimental loops consist of three aspects: 1) the intrinsic precision limit of the DFT calculations leads to small differences over different computational runs of the same organic-inorganic structure $(\sim 0.025(\mathrm{eV} / \mathrm{f} . \mathrm{u})$.$) , and 2)$ the uncertainty of the regression model that map the sparse DFT data over a continuous compositional space, and 3) the differences between simulation environment and experimental conditions due to kinetic factors.

\section{Data Integrity}

The synthesis conditions were calculated using Google Sheets, recorded using a laboratory notebook by the experimenter, then transcribed to a summary Google Sheets. Accelerated aging test data (camera images) were automatically pushed to Dropbox, and colour-calibration and averaging to sample-wise data was done by automated Python code. The Bayesian optimization codes were written using Python and gave the subsequent round's composition suggestions saved to a spreadsheet, which were then given to the experimenter, to be fabricated. Raw XRD, UV-Vis, SEM, GIWAXS data and JV data were processed/visualized using their own software packages with individual file format, labelling conventions, and stored on different local computers. Most of the data analysis was done using Python and MATLAB. Metadata, which linked different files containing synthesis conditions, calibrated aging test data, GIWAXS, XRD, and UV-Vis data were created on an ad-hoc basis on the 7 compositions, as samples were deemed of high scientific significance. 
Supplemental Figures
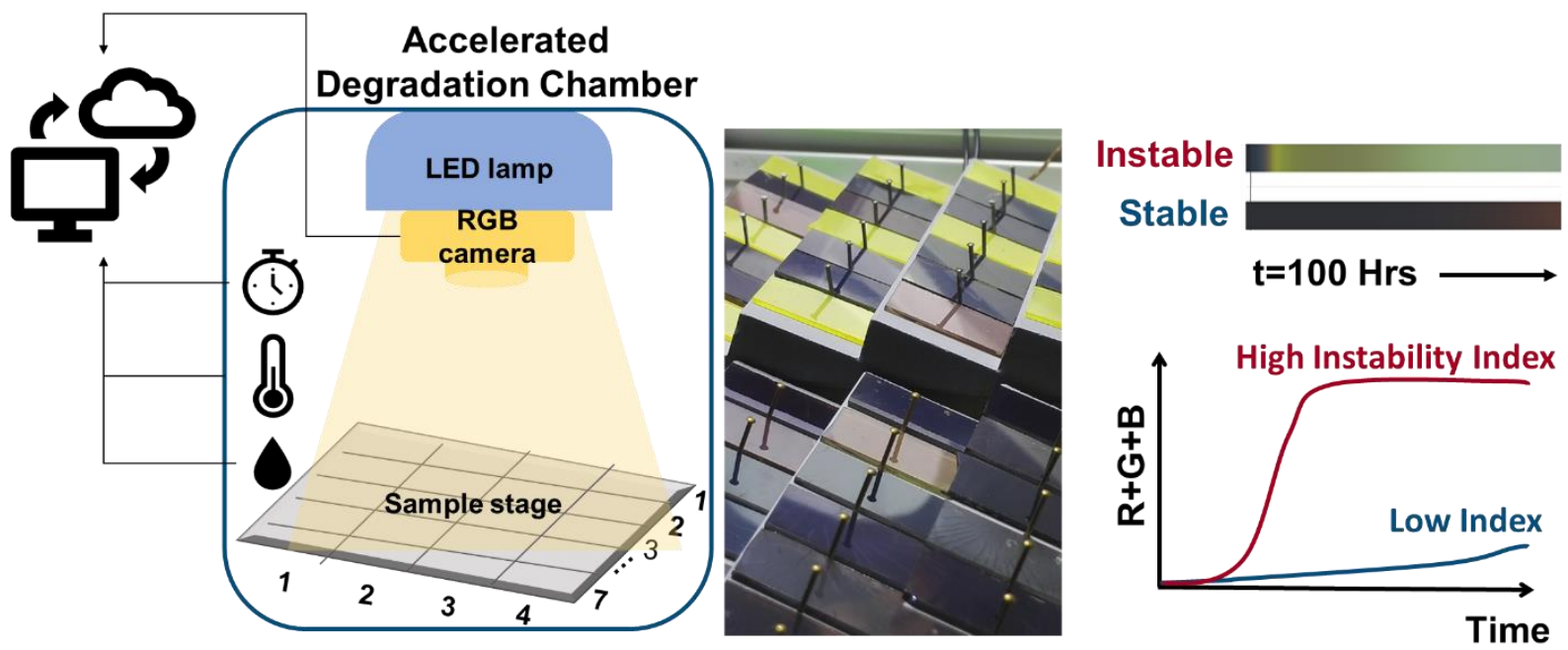

Figure S1, Schematics of the environmental chamber with in situ optical monitoring. 


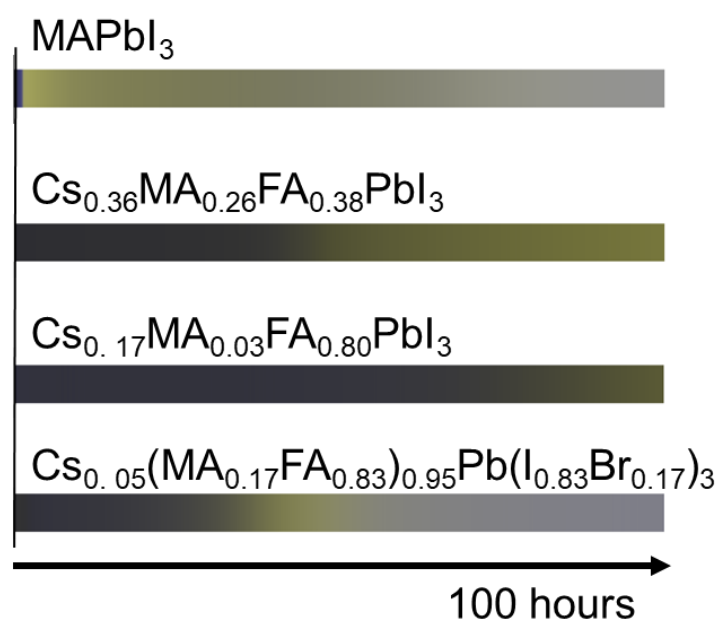

Figure S2. In situ monitored colour evolution of thin-films for four representative compositions during the degradation test, quantified $\mathrm{R}+\mathrm{G}+\mathrm{B}$ colour changes of the compositions are shown in Figure $3 \mathrm{~b}$ in the main article.

The decomposed and calibrated RGB values were determined for each degrading sample. This was evaluated across the film surface, and therefore resulted in a spatial distribution of colouration; this was evaluated by determining the mean, 5\% threshold, and 95\% threshold for each colour channel within each film. The upper and lower thresholds were utilized for confirming sufficient uniformity of the samples. The colour channels were evaluated separately, averaged, and utilized for calculating $I_{\mathrm{c}}$ for each sample according to the main article. In Figure $3 b$, the averaged colour channels were accumulated to yield an instability measure at each time point. This accumulation consisted of the sum of the R, G, and B mean values at a time point, normalized by the initial sum at zero minutes of degradation such that each sample had an instability measure of zero at the initial time recorded. In Fig, S2, the mean RGB was likewise visually represented as colour bars, relating the normalized average colour evaluated as a DI to an absolute recorded average colour over time. 


\section{Degradation Time}

6 Hours
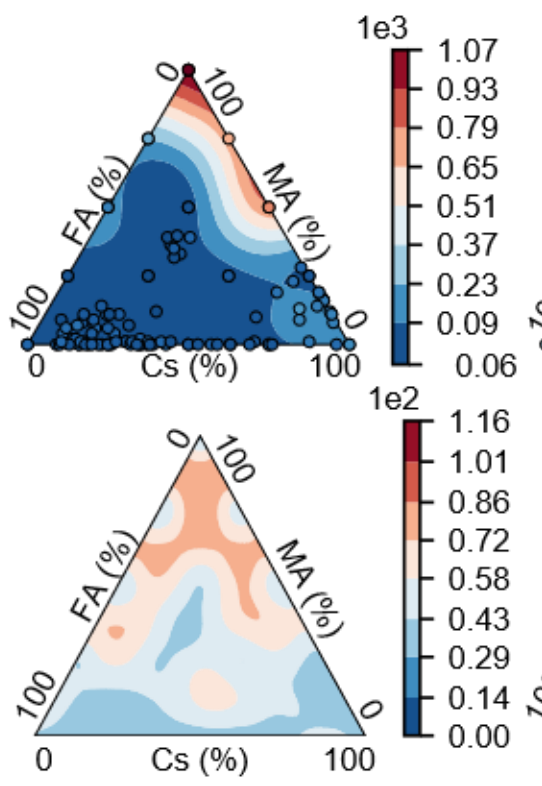

100 Hours
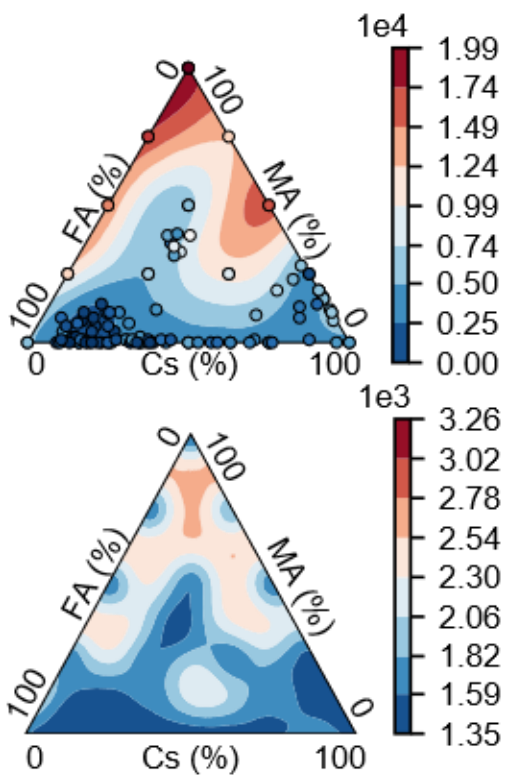

116.7 Hours

(7000 minutes full degradation test)

Figure S3. Integrated colour change over the first $6 \mathrm{~h}$ (left column), 100h (middle column), and 7000min (right column) of the aging test. 7000min result corresponds to the instability index. The resulting $I_{\mathrm{c}}$ (first row) and standard deviations (second row) are modelled using all the samples from rounds 0-3 and shown as contours. Each sample is shown as a dot with a colour corresponding to its experimentally measured $I_{\mathrm{c}}$.

Figure S3 illustrates the integrated colour changes and the uncertainties over the full compositional space using a GP model for three different integration (degradation test) periods. The results illustrate that after $6 \mathrm{~h}$ of degradation samples that do not have FA and have high MA content have clearly changed their colour, whereas other samples have gone through small, uniform colour changes. By 100h of degradation, the samples have already gone through most of the colour change seen at the final stage after 7000min, and the stable regions have separated from the rest of the space. The instability landscape derived from the colour change is consistent with the bandgap change trends (Figure S13), degradation products and structural changes measured by synchrotron-based GIWAXS at the same timepoints. GIWAXS (Figure S15) validates that mixed-cation perovskites have gone through minor degradation after 6 hours, leading to colour changes, and the most stable region I is not distinguishable from less stable ones. After 100 hours of degradation tests, the differences between the stable regions are visible through colour changes. The full list of samples and measured $I_{\mathrm{c}}$ are shown in Data. 
Round 0
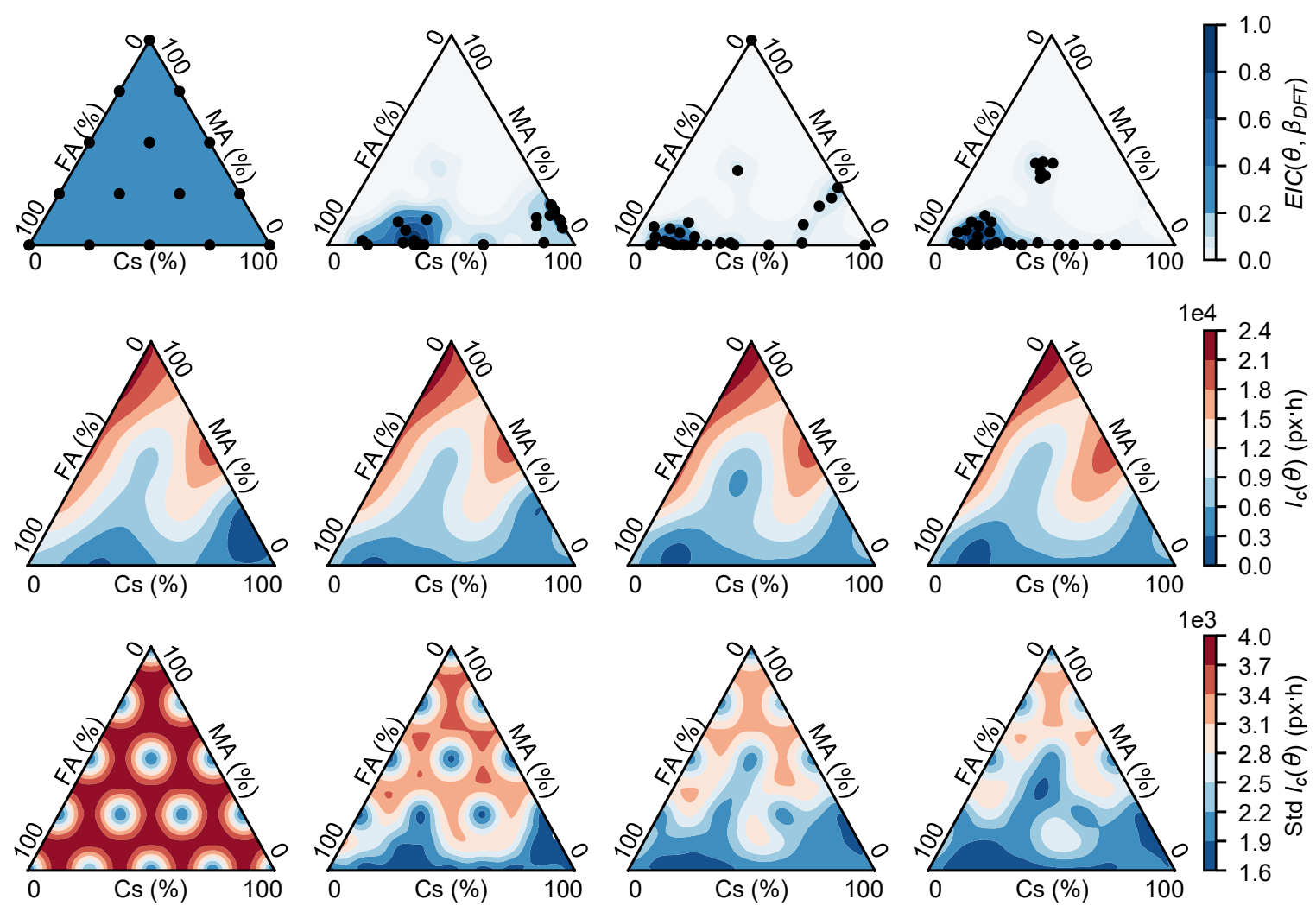

Figure S4. DFT-weighted acquisition function, posterior mean, and uncertainty landscapes over three optimization rounds.

Figure S4 presents all the rounds of Bayesian optimization, including the GP surrogate model mean and standard deviation, along with the DFT-weighted and unweighted acquisition functions. In Round 0, a grid of 28 evenly-spaced sample compositions (15 different compositions) was aged, and $I_{\mathrm{c}}$ was quantified for each composition. In each subsequent round, we use Bayesian optimization to efficiently sample compositions and iteratively approach the global optimum. The Bayesian optimization algorithm is implemented based on the GPyOpt optimization package. ${ }^{9}$ For our applications, we use a GP model with a Matérn 5/2 kernel as a surrogate model, with kernel parameters chosen to optimize the maximum likelihood. The GP model is updated in each experimental round until the search converges. Our Bayesian optimization has two convergence criteria: the modelled mean stabilized, and the instability index of the most stable compositions found reached the experimental uncertainty of our test setup. The convergence of the instability index towards the optimum is illustrated in Figure S4-5. 


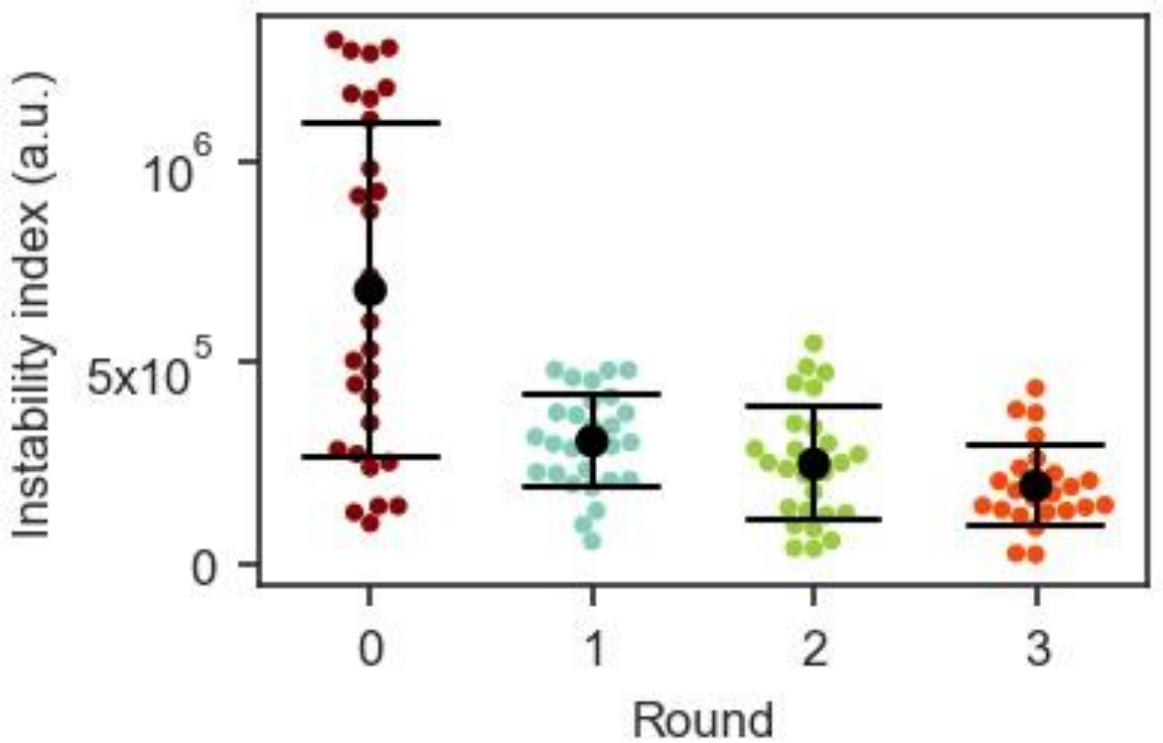

Figure S5. Instability index in logarithm scale for each degradation round with mean and standard deviation. 


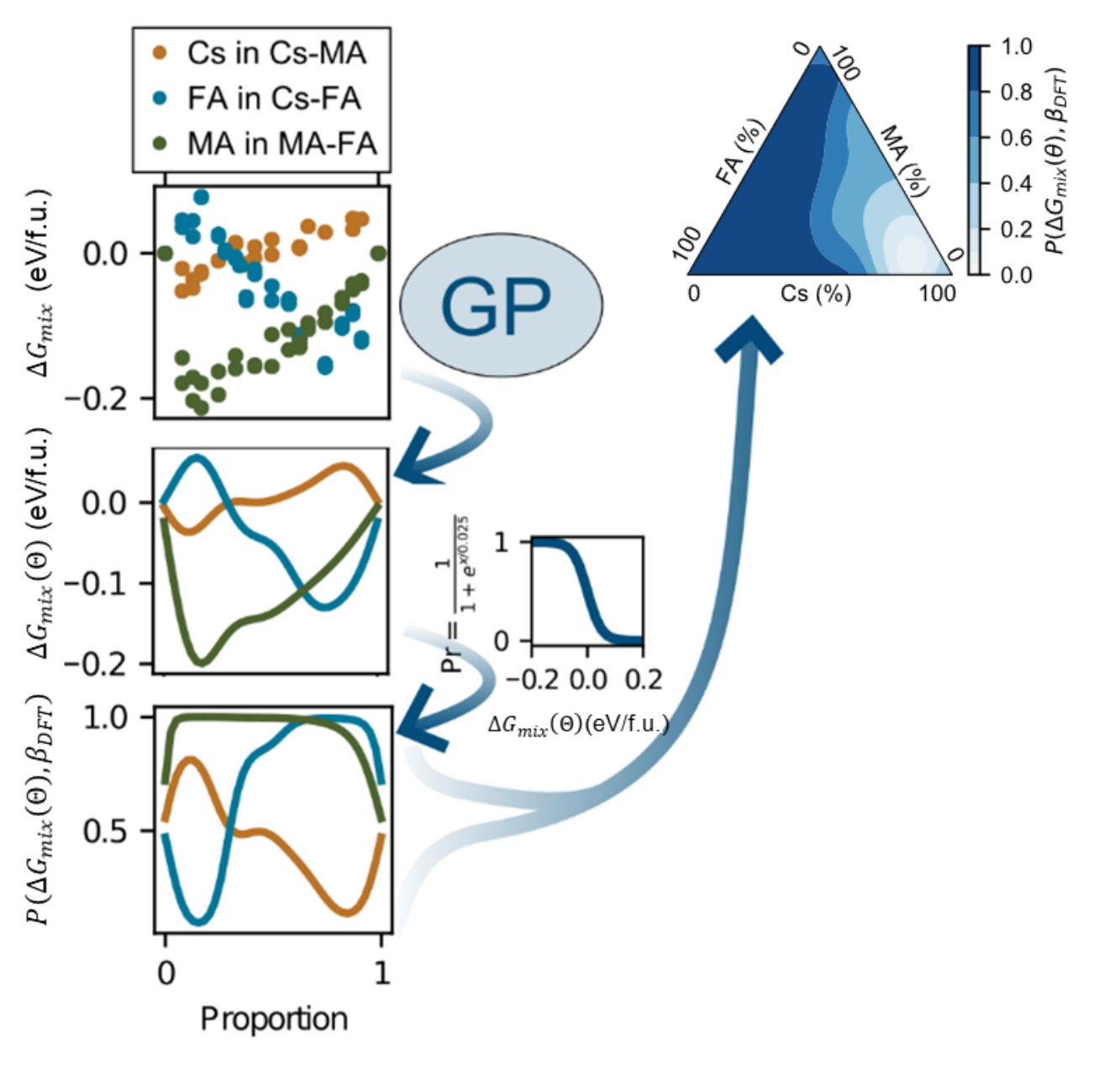

Figure S6. Construction of Gibbs free energy model using DFT-calculated Gibbs free energy of mixing. 
Acquisition prob. Acquisition prob. (with constraint) (no constraint)
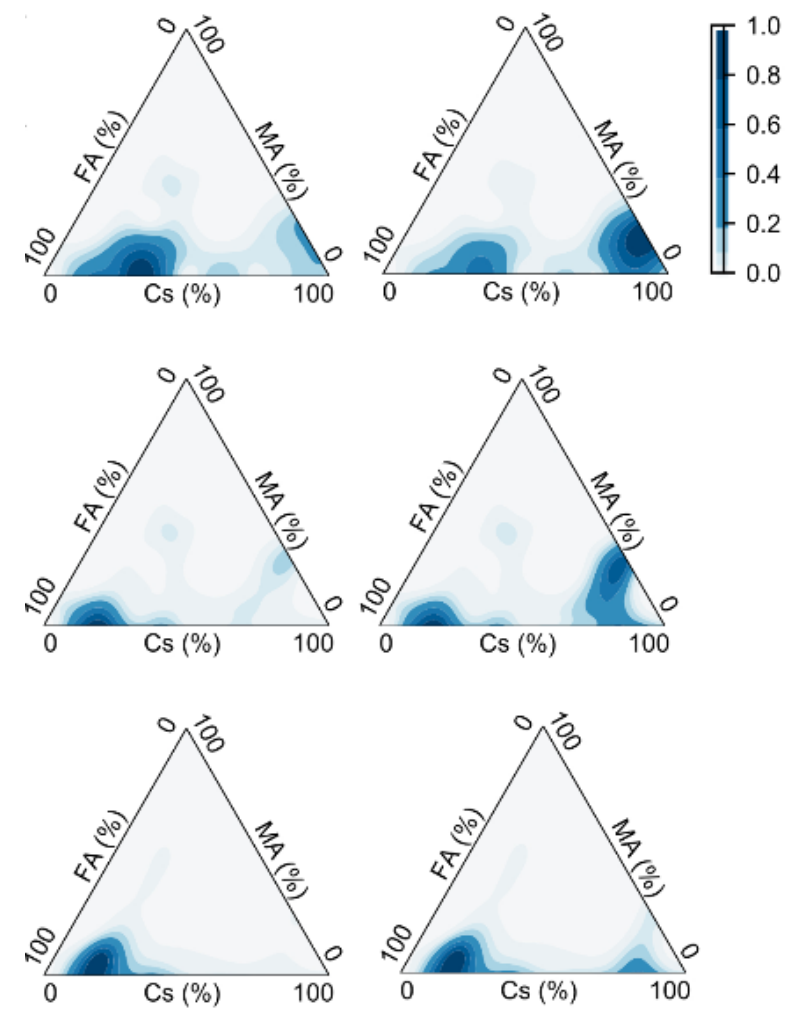

FigureS7. DFT-constrained acquisition function $(E I C)$ and unconstrained acquisition function (EI) shown after degradation rounds 0 (initialization, top), 1 (middle), and 3 (bottom). 


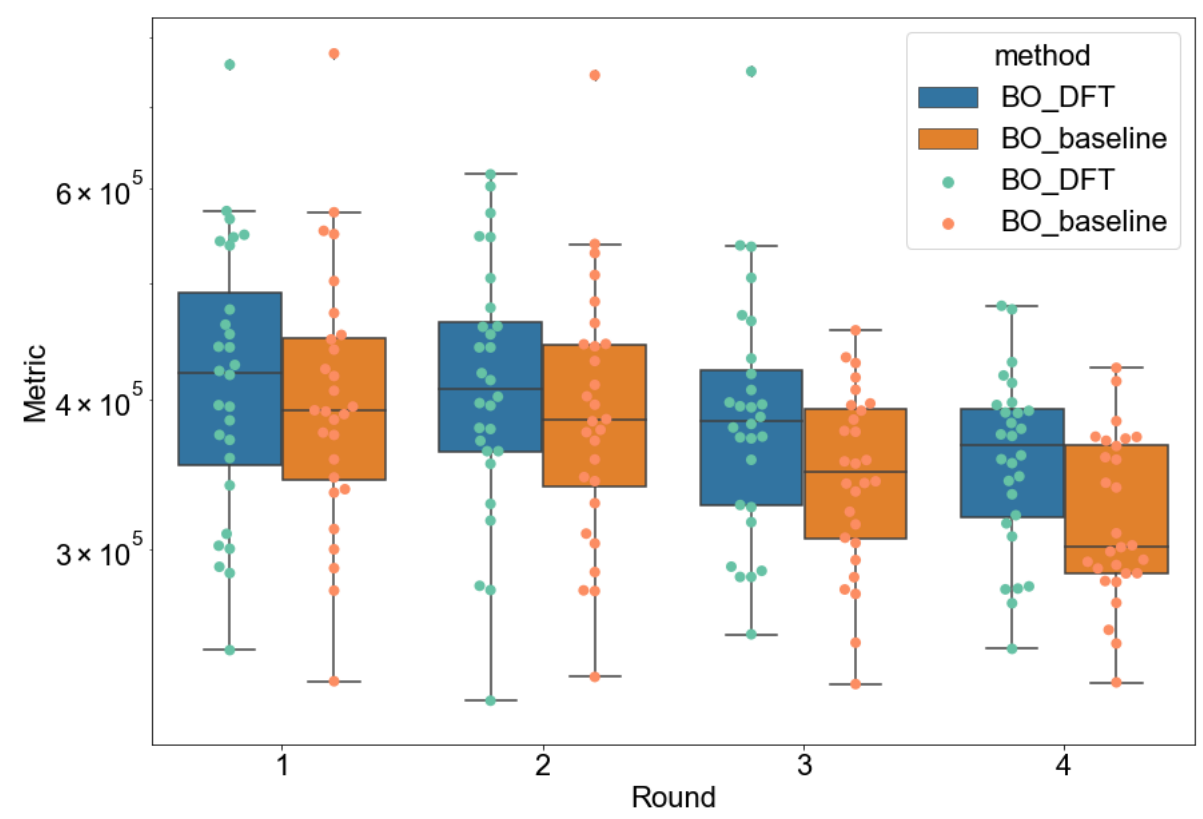

Figure S8. Loss evolution of student model, batch size $=28$

We use teacher-student model to compare the performance of Bayesian optimization fused with DFT data (BO_DFT) and standard Bayesian Optimization (BO_baseline). ${ }^{10}$ Firstly, we train a GP surrogate model as the "ground-truth" model and use it to calculate the instability index (metric) of newly suggested experimental conditions from both BO with DFT constraint (BO_DFT) and BO without DFT constraint (BO_baseline). We feed the results of the initial run (run 0) to the above two different Bayesian optimization methods and suggest new conditions iteratively from run 1 to run 4 , in order to simulate our experimental optimisation rounds. The teacher-student model allows comparison of those two methods without performing the actual time-consuming degradation experiments. Figure S8 shows the loss evolution of the student model with batch size of 28. The median loss decreases for both DFT constrained and unconstrained BO. Unconstrained BO has lower instability metric than DFT constrained because the former one allocates most of points in the lower right corner of the phase diagram (Figure S9). This region shows phase demixing before degradation from the XRD measurement (Figure S11) despite minimal colour change of films in this region during degradation. DFT constrained BO avoids exploring that area with DFT data fusion and finds the local minimum at lower left corner of the phase diagram. 


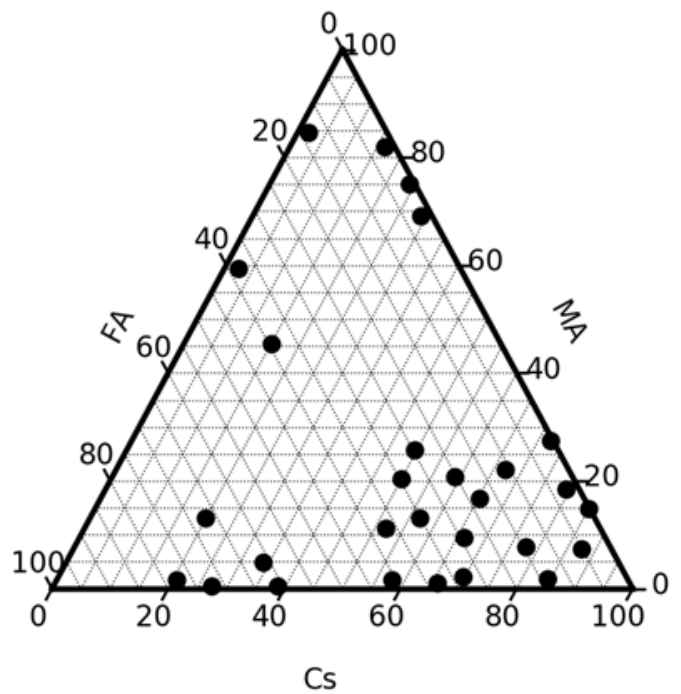

Suggestions without Data Fusion

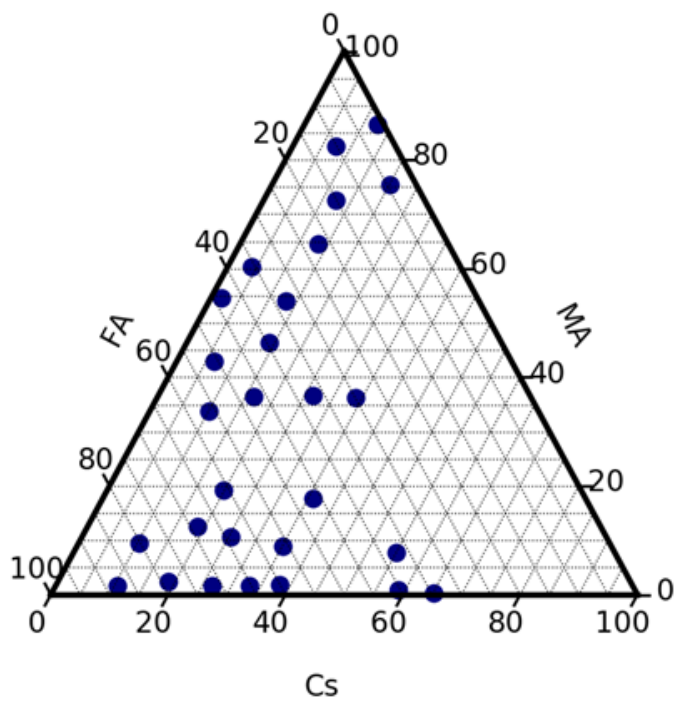

Suggestions with Data Fusion

Figure S9. Suggested points of student model for Round 4, batch size $=28$ 


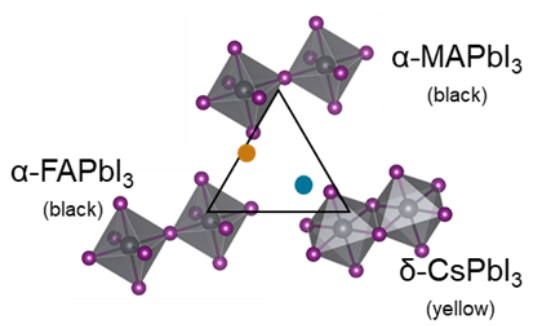

Crystal structures at $403 \mathrm{~K}$

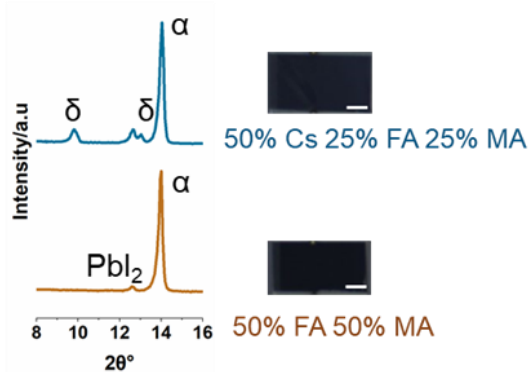

As-synthesized Films

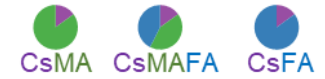

$\delta-\mathrm{FAPbl}_{3} \delta-\mathrm{CsPbl}_{3}$

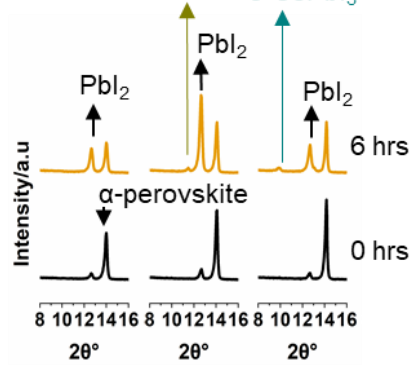

Mechanistic role of MA

Figure S10. Crystal structures of the end members of the $\mathrm{Cs}_{\mathrm{x}} \mathrm{MA}_{y} \mathrm{FA}_{1-\mathrm{x}-\mathrm{y}} \mathrm{PbI}_{3}$ phase space at thin-film synthesis temperature. Minority phases are detected via grazing incidence X-ray diffraction measurement on as-synthesized thin film samples. Over-stoichiometric lead iodide was added in the synthesis following reference, ${ }^{1}$ leading to $\mathrm{PbI}_{2}$ crystallization in as-synthesized films. Scale bar: 0.2 inches. Mechanistic role of MA in hot and humid conditions: X-ray diffraction patterns of perovskites after 6 hours' degradation test, with fixed $\mathrm{Cs}$ content. CsMA: $\mathrm{Cs}_{0.15} \mathrm{MA}_{0.85} \mathrm{PbI}_{3}$. CsFA: $\mathrm{Cs}_{0.15} \mathrm{FA}_{0.85} \mathrm{PbI}_{3} \mathrm{CsMAFA}$ $\mathrm{Cs}_{0.15} \mathrm{MA}_{0.425} \mathrm{FA}_{0.425} \mathrm{PbI}_{3}$. 


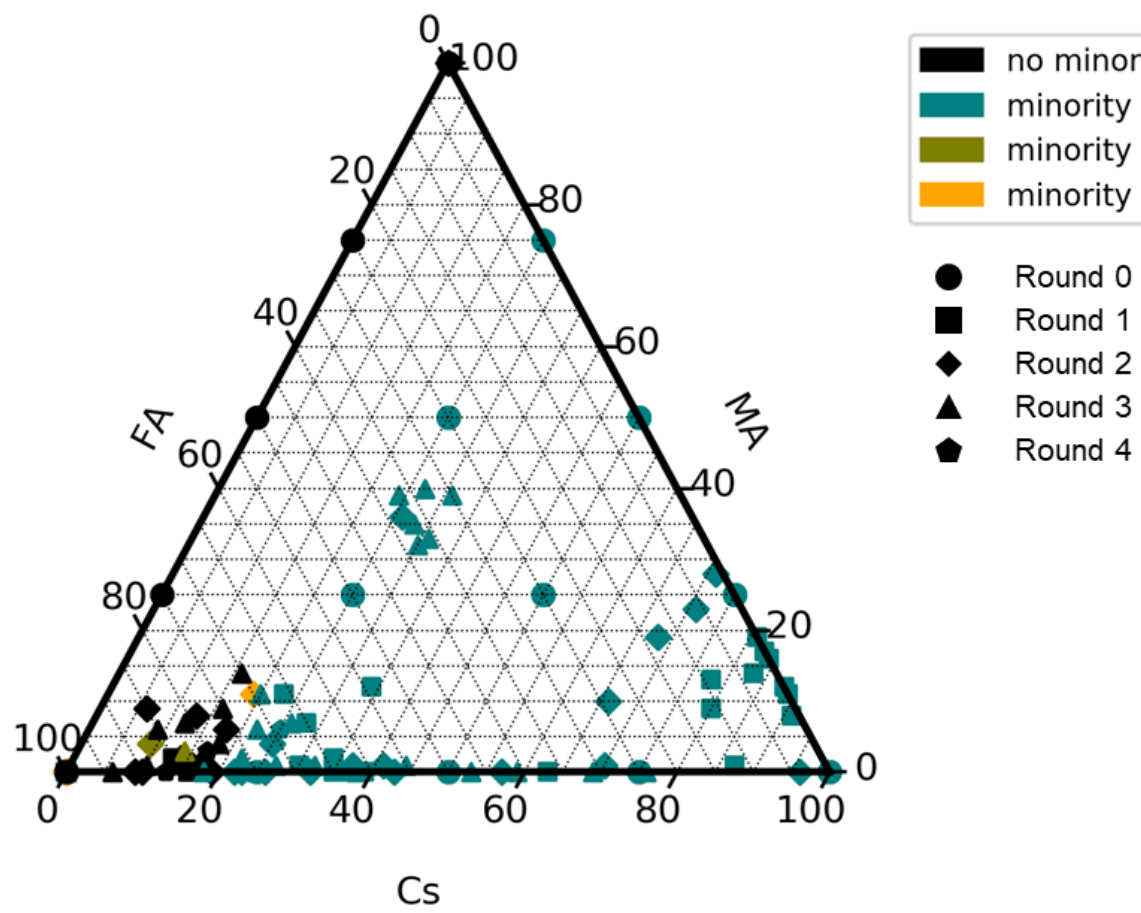

Figure S11. Phase diagrams constructed using XRD measurements. No minority phases indicate singlephase $\alpha$-perovskite, minority phase $\mathrm{Cs}$ indicates the existence of $\delta$-CsPbI ${ }_{3}$, minority phase FA indicates the existence of $\delta-\mathrm{FAPbI}_{3}$ crystalized at $403 \mathrm{~K}$ thin-film annealing temperature followed by cooling to $300 \mathrm{~K}$ room temperature in $\mathrm{N}_{2}$ glovebox prior to degradation tests. Full list of X-ray diffraction patterns is shown in Supplementary Data. 


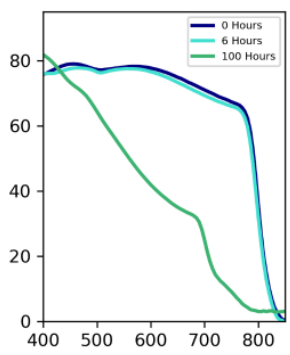

a

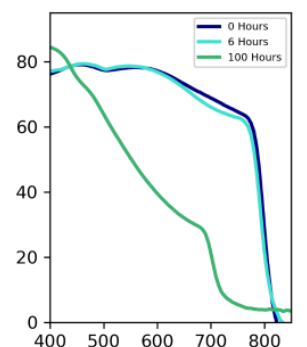

b

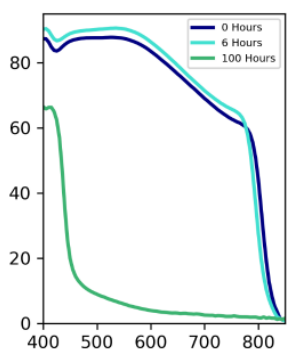

c

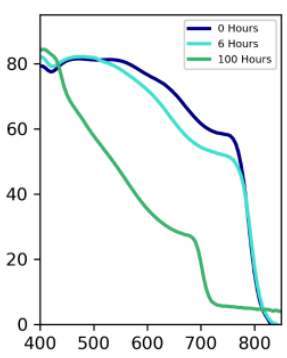

d

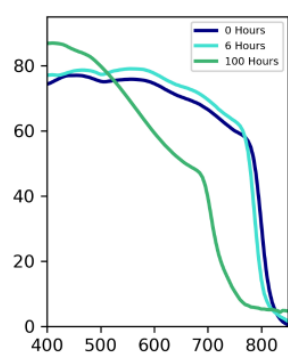

$\mathrm{e}$

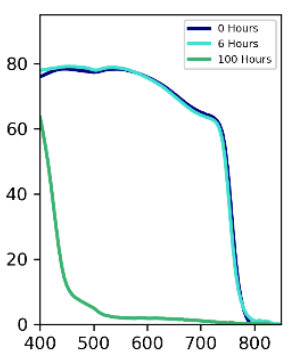

f

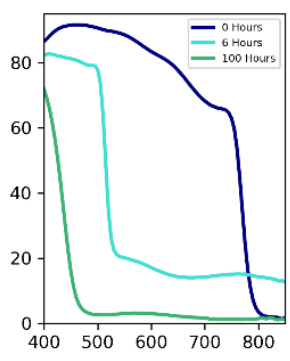

$\mathrm{g}$

Figure S12. Absorption spectra of composition a-g, dark blue: as synthesized, cyan: after degradation for 6 hours, green: after degradation for 100 hours. Composition ID a-g following Table S2. 

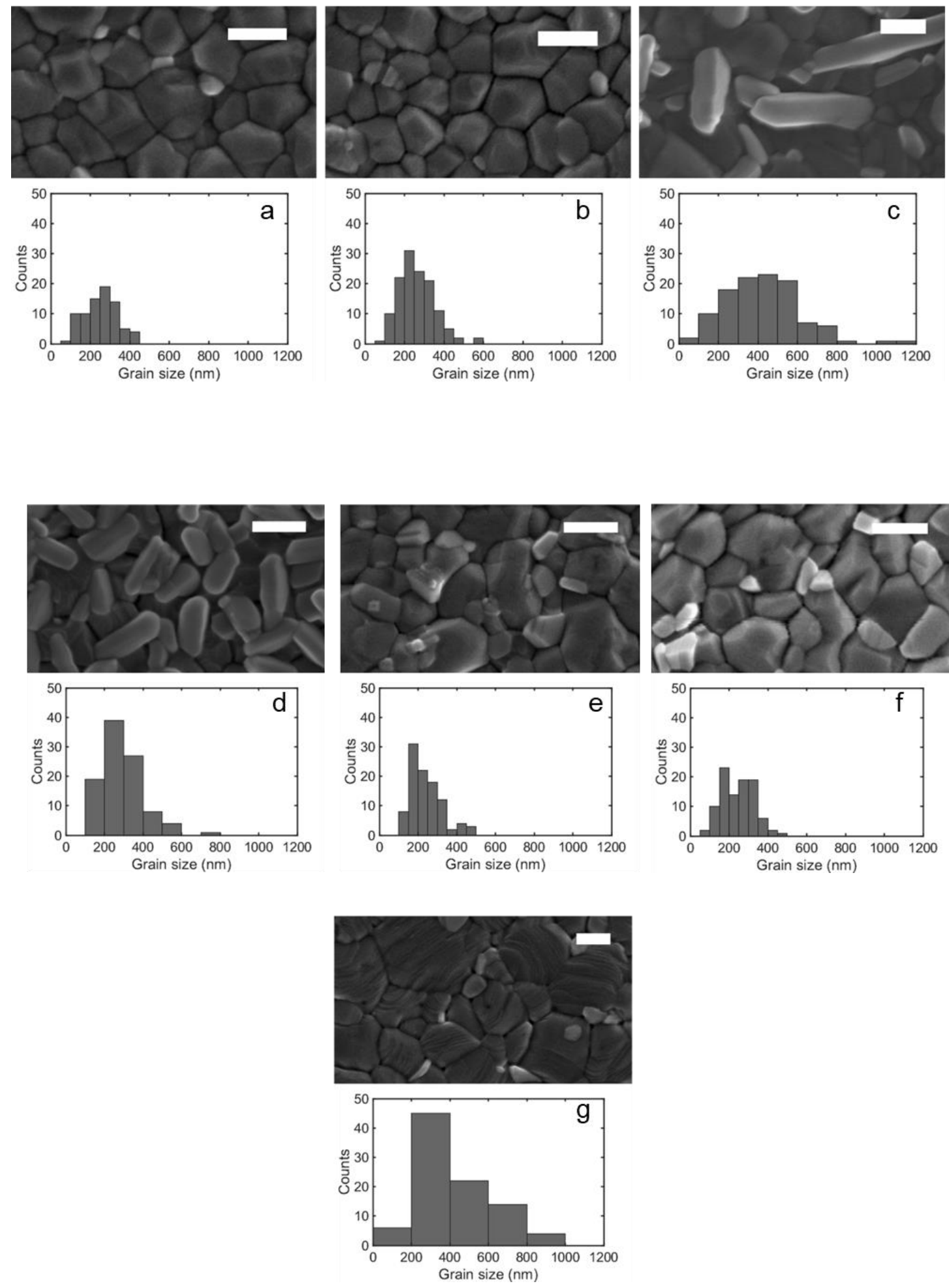

Figure S13. SEM images and grain size count for as-synthesized composition a-g in Round 4. The scale bar is $300 \mathrm{~nm}$. Rod-like $\delta-\mathrm{CsPbI}_{3}$ minority phases are visible in c and d. Composition ID a-g following Table S2. 


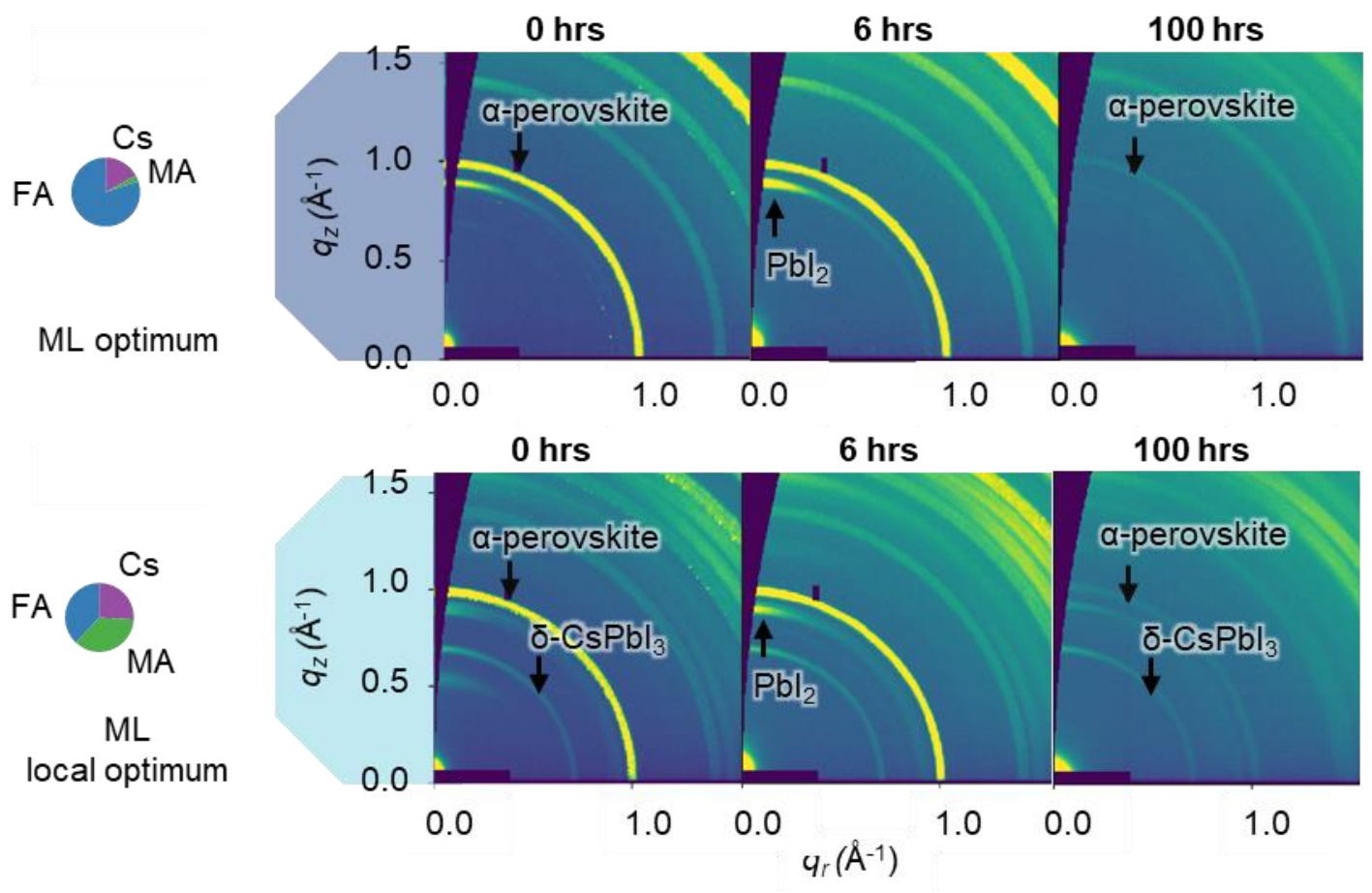

Figure S14. GIWAXS images with data acquisition at incident angle of $\theta=0.2^{\circ}$ for composition d (MLlocal optimum) and e (ML local optimum) after 0, 6, and, 100 hours of degradation tests. Composition ID follows Table S4. These images represent the raw data collection for Figure $4 \mathrm{c}$ and $\mathrm{f}$ in the main text. 

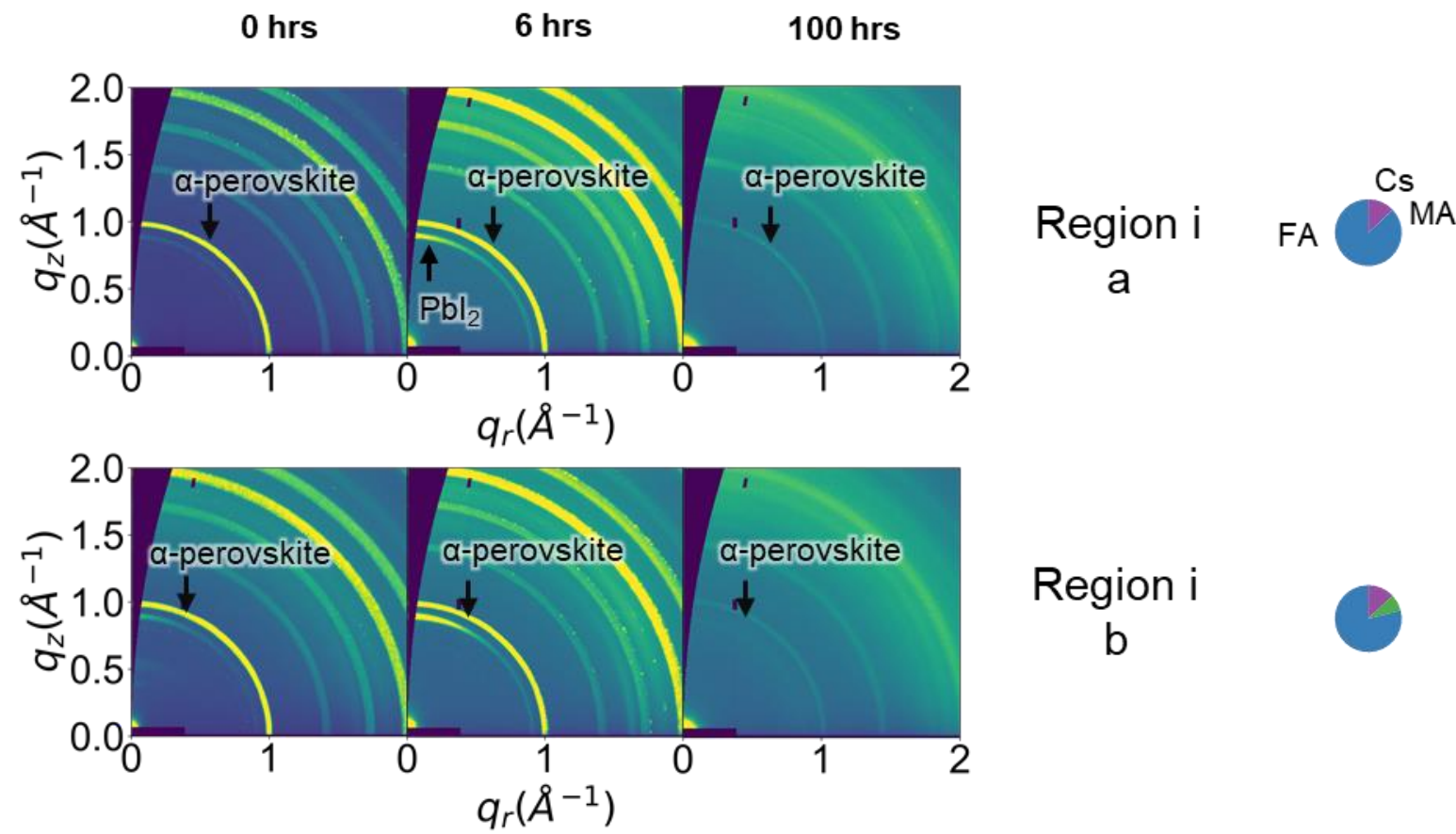

Region i

b

Region ii

C

\section{Region iii} $\mathrm{f}$

Figure S15. GIWAXS images with data acquisition at incident angle of $\theta=0.2^{\circ}$ for composition $a, b, c$, and $\mathrm{f}$ after 0, 6 (Round 5), and, 100 hours (Round 6) of degradation tests. Composition ID follows Table S4. The measurements on composition $b$ and $c$ represent the raw data collection for Figure $4 \mathrm{~d}$ and e, respectively. 

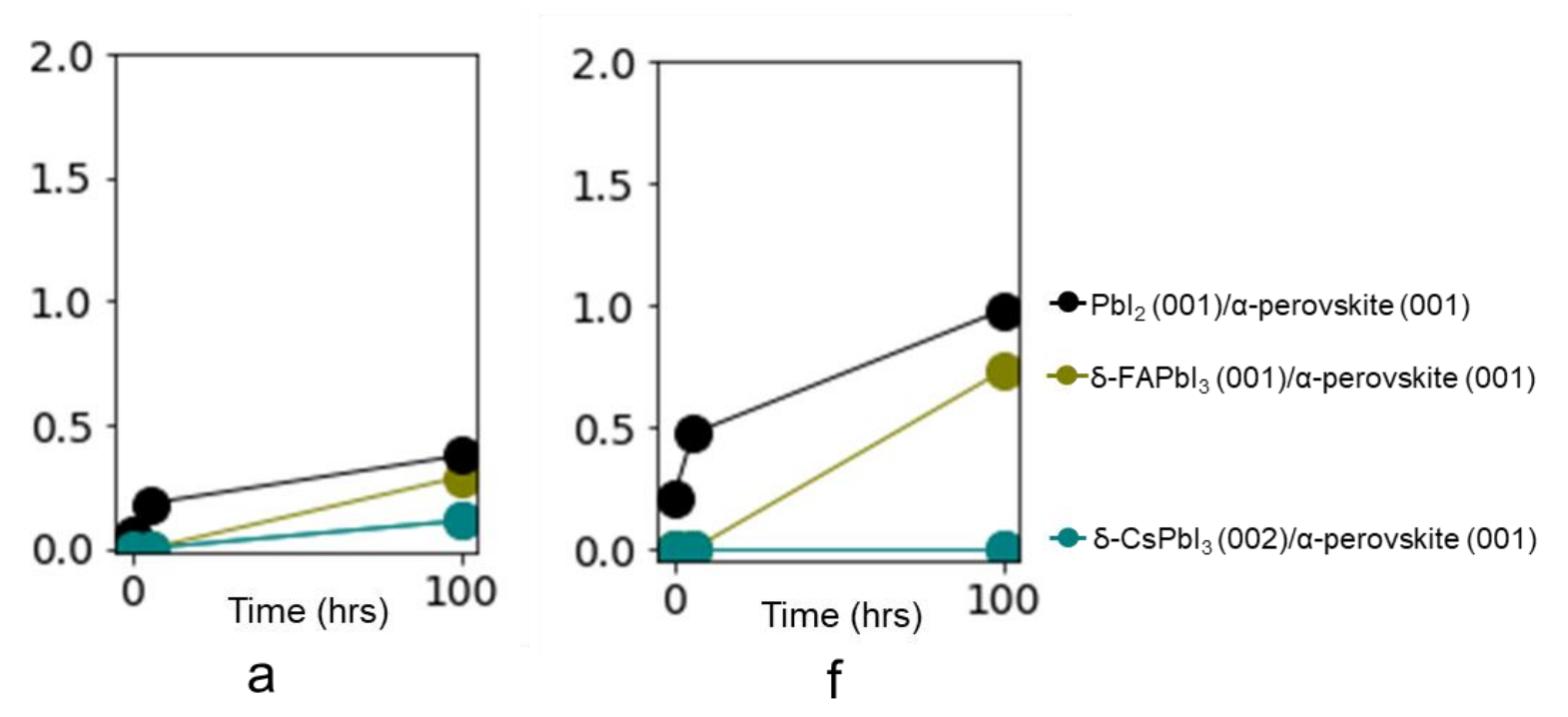

Figure S16. Relative peak insensitive of GIWAXS data (circular average) for composition a and $\mathrm{f}$ after 0, 6 (Round 5), and, 100 hours (Round 6) of degradation tests. Composition ID follows Table S4. Figures for composition b-e are shown in main text Figure 4 e-h. 


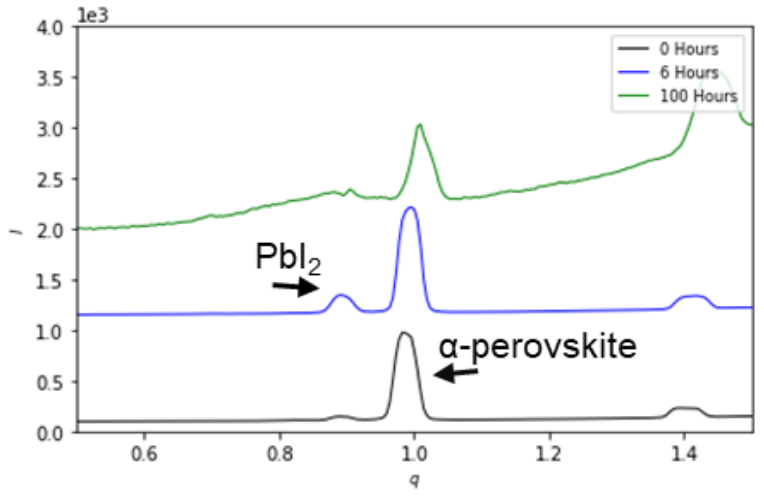

a

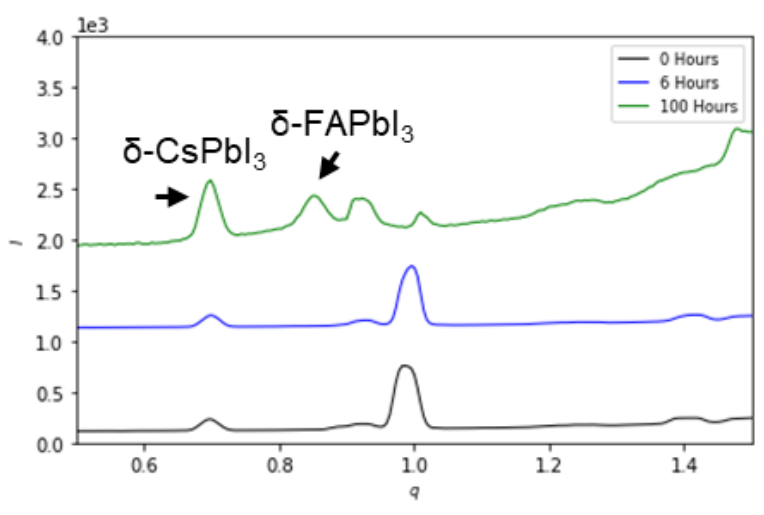

C

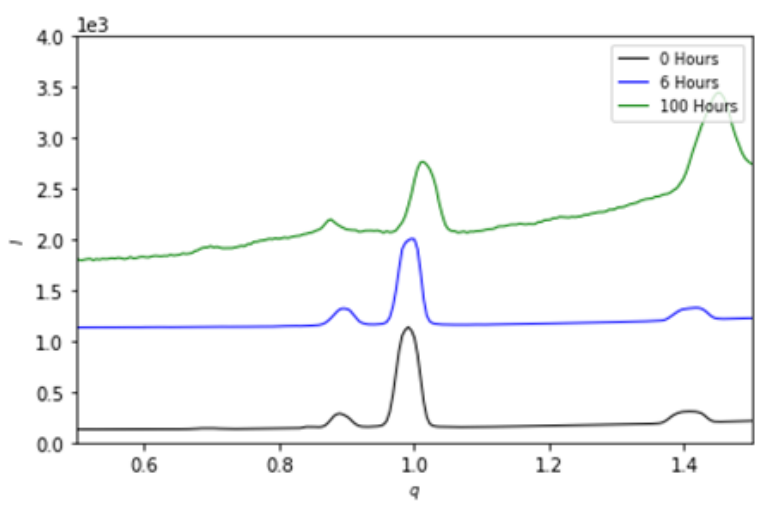

e

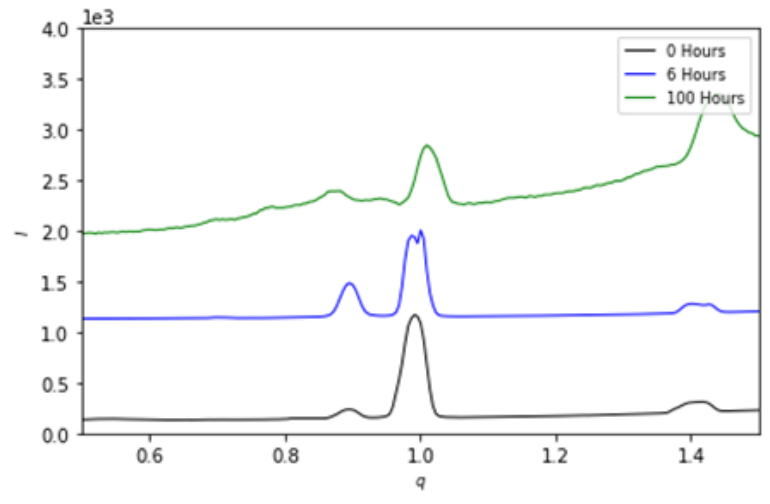

b

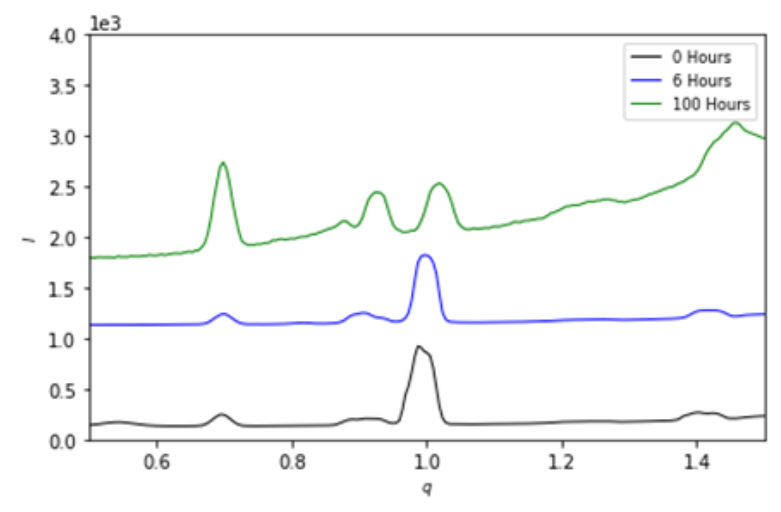

d

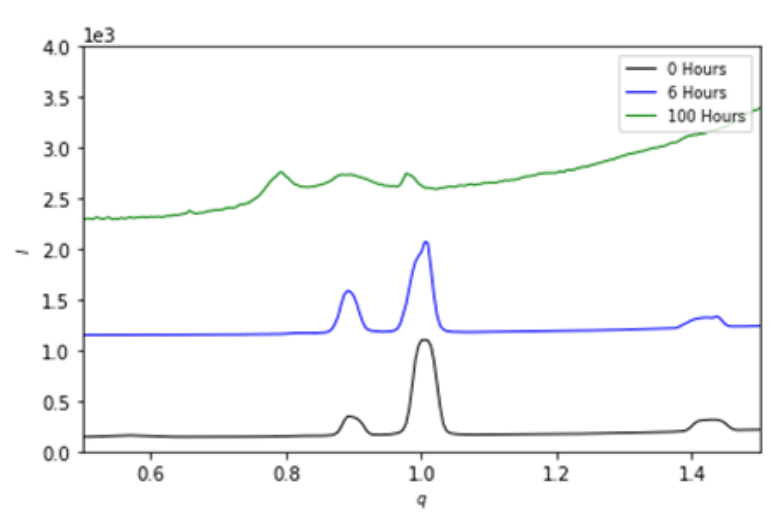

f

Figure S17. Integrated peak intensities for compositions a, b, c, d, e, and f after 0,6 , and 100 hours of degradation tests respectively. We observe the emergence of $\mathrm{PbI}_{2}, \delta-\mathrm{CsPI}_{3}$ and $\delta-\mathrm{FAPbI}_{3}$ during the course of degradation test. We observe a lattice shrinkage for all the iodide-based perovskites, including both CsFA, and CsMAFA perovskites. 

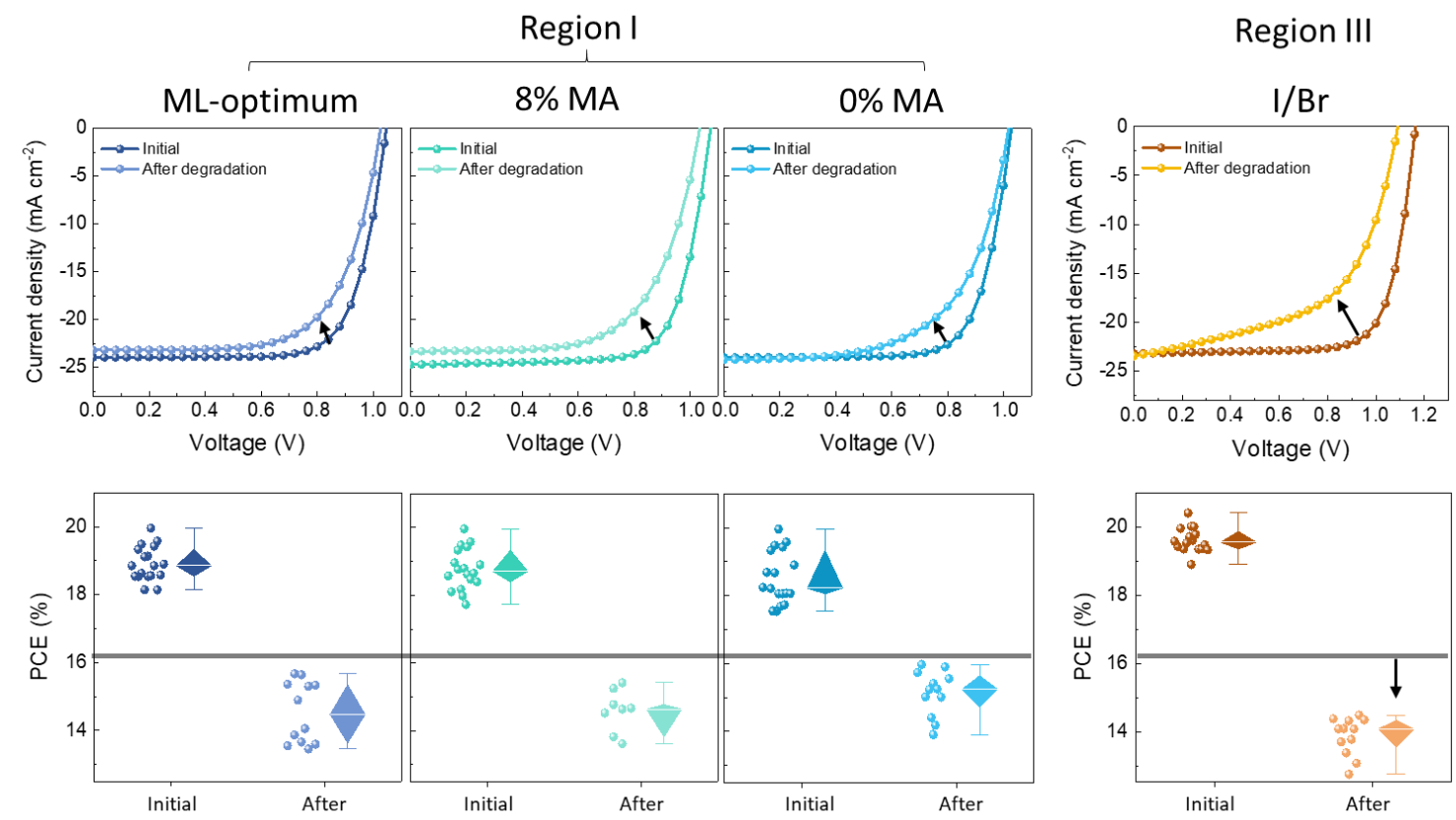

Figure S18. $J-V$ curves before and after degradation. Aging conditions: unencapsulated 50 hours under $85 \% \mathrm{RH} / 85^{\circ} \mathrm{C}$ in air under dark. The horizontal lines and arrows are for eye guidance only. 


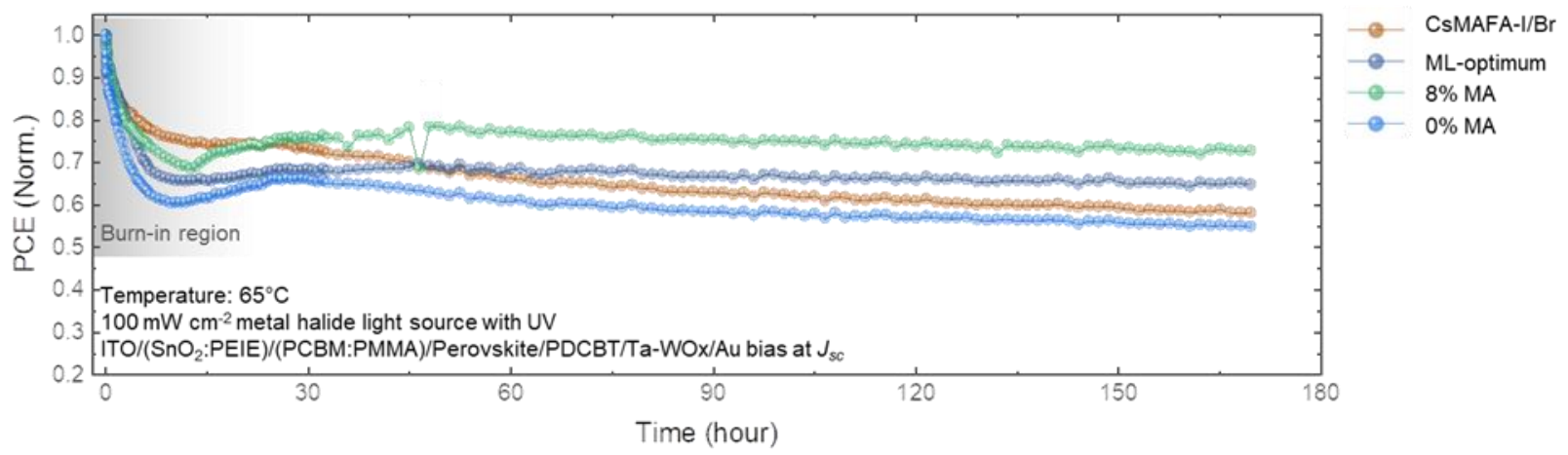

Figure S19 Time-resolved individual device performance of optimized iodide perovskites and $\mathrm{I} / \mathrm{Br}$ mixed anion perovskite composition. Aging conditions: unencapsulated under $65^{\circ} \mathrm{C}$ in $\mathrm{N}_{2}$ under 1 Sun. Burn-in region: initial efficiency drop within the first 20 hours attributed to interfacial mechanisms depending on device archetectures. ${ }^{8}$ 


\section{Supplemental Tables}

Table S1. Summary of sample synthesis and degradation tests.

\begin{tabular}{|c|c|c|c|c|c|c|c|}
\hline $\begin{array}{c}\text { Synthesis } \\
\text { Round }\end{array}$ & $\begin{array}{c}\text { No. of samples } \\
\text { synthesized }\end{array}$ & No. of compositions & $\begin{array}{c}\text { Full or partial } \\
\text { degradation round }\end{array}$ & $\begin{array}{c}\text { Structure } \\
\text { Characterization }\end{array}$ & Sampling strategies & $\begin{array}{l}\text { No. of triple cation } \\
\text { compositions }\end{array}$ & $\begin{array}{c}\text { No. of single or } \\
\text { double cation } \\
\text { composition }\end{array}$ \\
\hline 0 & 30 & 15 & Full & XRD & Equal-spaced sampling & 3 & 12 \\
\hline 1 & 28 & 21 & Full & XRD & ML suggests with DFT & 12 & 16 \\
\hline $1 \mathrm{~b}$ & 28 & 28 & Full & XRD & ML suggests without DFT & 10 & 18 \\
\hline 2 & 28 & 28 & Full & XRD & $\begin{array}{l}\text { ML suggests with DFT, } 1 \text { manually added (two } \\
\text { compositions are within } 1 \% \text { differences) }\end{array}$ & 18 & 10 \\
\hline 3 & 32 & 32 & Full & XRD & $\begin{array}{l}\text { ML suggests with DFT, } 4 \text { added manually } \\
\text { (validation of local optimum) }\end{array}$ & 21 & 11 \\
\hline 4 & 28 & 7 & Full & XRD & $\begin{array}{c}\text { Selected } 7 \text { representative compositions, } \\
\text { validation round }\end{array}$ & 4 & 3 \\
\hline 5 & 14 & 7 & 6 hours & GIWAXS & $\begin{array}{l}\text { Samples degraded up to a time point for } \\
\text { synchrotron measurement. }\end{array}$ & 4 & 3 \\
\hline 6 & 14 & 7 & 100 hours & GIWAXS & $\begin{array}{l}\text { Samples degraded up to a time point for } \\
\text { synchrotron measurement. }\end{array}$ & 4 & 3 \\
\hline
\end{tabular}


Table S2. List of 7 representative compositions selected for advanced structural, optical and morphological characterization.

\begin{tabular}{|l|l|l|l|l|l|}
\hline Stability Region & Composition ID & Cs\% & MA\% & FA\% & Reason for in-depth characterization \\
\hline I & a & 13 & 0 & 87 & Top binary cations Round 0 -3 \\
\hline I & b & 13 & 8 & 79 & Top binary cations Round 0 -3 \\
\hline II & c & 26 & 74 & 0 & Reference composition with no MA \\
\hline II & d & 26 & 38 & 36 & ML local optimum \\
\hline I & e & 17 & 3 & 80 & ML optimum \\
\hline III & f & 5 & 16.15 & 78.85 & Reference composition, state of the art \\
\hline IV & g & 0 & 100 & 0 & Reference composition with no FA or Cs \\
\hline
\end{tabular}


Table S3. Bandgap changes after degradation tests.

\begin{tabular}{|l|l|l|l|l|l|l|l|}
\hline \multicolumn{7}{|l|}{ Bandgap (eV)* } & \multicolumn{2}{l|}{} \\
\hline Time (hours) & a & b & c & d & e & f & g \\
\hline 0 & 1.48 & 1.5 & 1.48 & 1.5 & 1.49 & 1.58 & 1.55 \\
\hline 6 & 1.48 & 1.5 & 1.49 & 1.5 & 1.51 & 1.58 & 2.28 \\
\hline 100 & 1.61 & 1.64 & 2.61 & 1.67 & 1.61 & 2.64 & 2.6 \\
\hline
\end{tabular}

*Estimated error of $\pm 0.03 \mathrm{eV}$. 
Table S4. Sample list for GIWAXS examination. The Composition ID follows the ID in Table S1.

\begin{tabular}{|l|l|l|l|l|l|}
\hline Stability Region & Composition ID & Cs\% & MA\% & FA\% & $\begin{array}{l}\text { Duration of } \\
\text { Degradation } \\
\text { (Hours) }\end{array}$ \\
\hline I & a & & & & $0,6,100$ \\
\hline I & b & 13 & 0 & 87 & $0,6,100$ \\
\hline II & c & 13 & 8 & 79 & $0,6,100$ \\
\hline II & d & 26 & 74 & 0 & $0,6,100$ \\
\hline I & e & 26 & 38 & 36 & $0,6,100$ \\
\hline III & f & 17 & 3 & 80 & $0,6,100$ \\
\hline
\end{tabular}


Table S5. Peak index from GIWAXS images

\begin{tabular}{|c|c|c|c|c|c|c|c|c|c|c|c|c|c|c|c|c|}
\hline \multirow[t]{3}{*}{$\begin{array}{l}0 \\
\text { Hours }\end{array}$} & & \multicolumn{10}{|c|}{ Peak Positions $(q)$ and Intensities (I) (Circular Average) } & \multicolumn{5}{|c|}{ Background-subtracted } \\
\hline & \multirow{2}{*}{$\begin{array}{l}\text { Background } \\
I\end{array}$} & \multicolumn{2}{|c|}{ Perovskite (001) } & \multicolumn{2}{|c|}{$\mathrm{CsPb}_{3}(002)$} & \multicolumn{2}{|c|}{$\mathrm{FAPbI}_{3}(001)$} & \multicolumn{2}{|c|}{$\mathrm{PbI}_{2}(001)$} & \multicolumn{2}{|c|}{$\mathrm{CsPb}_{3}(102)$} & \multirow{2}{*}{$\begin{array}{l}\text { Perovskite } \\
I\end{array}$} & \multirow{2}{*}{$\begin{array}{l}\mathrm{CsPb}_{3} \\
(002) \\
I\end{array}$} & \multirow{2}{*}{$\begin{array}{l}\mathrm{FAPbI}_{3} \\
(001) \\
I\end{array}$} & \multirow{2}{*}{$\begin{array}{l}\mathrm{PbI}_{2} \\
(001) \\
I\end{array}$} & \multirow{2}{*}{$\begin{array}{l}\mathrm{CsPbI}_{3} \\
(102) \\
I\end{array}$} \\
\hline & & $Q$ & $I$ & $Q$ & $I$ & $q$ & $I$ & $Q$ & $I$ & $q$ & $I$ & & & & & \\
\hline A & 97.288 & 0.982 & 976.143 & 0.707 & 102.043 & 0.840 & 109.921 & 0.891 & 145.630 & & & 878.855 & & 12.633 & 48.342 & \\
\hline B & 136.883 & 0.991 & 1170.167 & 0.707 & 135.709 & 0.845 & 147.407 & 0.895 & 236.317 & & & 1033.284 & & 10.524 & 99.434 & \\
\hline $\mathrm{C}$ & 115.909 & 0.987 & 759.443 & 0.707 & 200.604 & 0.845 & 128.772 & 0.900 & 163.261 & 0.932 & 184.984 & 643.534 & 84.695 & 12.863 & 47.352 & 69.075 \\
\hline $\mathrm{D}$ & 132.751 & 0.991 & 911.080 & 0.707 & 209.716 & 0.849 & 138.121 & 0.895 & 196.309 & 0.987 & 204.799 & 778.329 & 76.965 & 5.370 & 63.558 & 72.049 \\
\hline $\mathrm{E}$ & 128.968 & 0.991 & 1137.794 & 0.707 & 138.190 & 0.849 & 155.693 & 0.891 & 284.678 & & & 1008.826 & & 26.725 & 155.709 & \\
\hline $\mathrm{F}$ & 145.045 & 1.005 & 1102.730 & 0.707 & 144.083 & 0.845 & 154.400 & 0.895 & 345.547 & & & 957.685 & & 9.355 & 200.502 & \\
\hline \multirow[t]{3}{*}{$\begin{array}{l}6 \\
\text { Hours }\end{array}$} & & \multicolumn{10}{|c|}{ Peak Positions $(q)$ and Intensities (I) (Circular Average) } & \multicolumn{5}{|c|}{ Background-subtracted } \\
\hline & Background & \multicolumn{2}{|c|}{ Perovskite (001) } & \multicolumn{2}{|c|}{$\mathrm{CsPbI}_{3}(002)$} & \multicolumn{2}{|c|}{$\mathrm{FAPbI}_{3}(001)$} & \multicolumn{2}{|c|}{$\mathrm{PbI}_{2}(001)$} & \multicolumn{2}{|c|}{$\mathrm{CsPbI}_{3}(102)$} & Perovskite & $\begin{array}{l}\mathrm{CsPbI}_{3} \\
(002)\end{array}$ & $\begin{array}{l}\mathrm{FAPbI}_{3} \\
(001)\end{array}$ & $\begin{array}{l}\mathrm{PbI}_{2} \\
(001)\end{array}$ & $\begin{array}{l}\mathrm{CsPbI}_{3} \\
(102)\end{array}$ \\
\hline & $I$ & $Q$ & $I$ & $Q$ & $I$ & $q$ & $I$ & $Q$ & $I$ & $q$ & $I$ & $I$ & $I$ & $I$ & $I$ & $I$ \\
\hline A & 152.982 & 0.996 & 1215.836 & 0.707 & 161.419 & 0.845 & 169.747 & 0.891 & 346.907 & & & 1062.854 & 8.437 & 16.765 & 193.925 & \\
\hline B & 132.009 & 1.001 & 1006.776 & 0.707 & 145.289 & 0.845 & 149.510 & 0.895 & 484.918 & & & 874.767 & 13.280 & 17.501 & 352.909 & \\
\hline $\mathrm{C}$ & 136.276 & 0.996 & 742.240 & 0.707 & 232.101 & 0.845 & 149.960 & 0.900 & 168.679 & 0.927 & 206.762 & 605.963 & 95.825 & 13.684 & 32.403 & 70.486 \\
\hline $\mathrm{D}$ & 132.035 & 0.996 & 822.620 & 0.707 & 217.400 & 0.845 & 145.112 & 0.900 & 242.712 & 0.927 & 205.790 & 690.585 & 85.365 & 13.077 & 110.677 & 73.755 \\
\hline $\mathrm{E}$ & 133.970 & 0.996 & 1006.303 & 0.707 & 141.311 & 0.845 & 150.138 & 0.895 & 320.102 & & & 872.333 & 7.341 & 16.168 & 186.132 & \\
\hline
\end{tabular}




\begin{tabular}{|c|c|c|c|c|c|c|c|c|c|c|c|c|c|c|c|c|}
\hline $\mathrm{F}$ & 149.877 & 1.005 & 1070.753 & 0.707 & 154.771 & 0.845 & 169.093 & 0.891 & 587.316 & & & 920.876 & 4.894 & 19.216 & 437.439 & \\
\hline $\begin{array}{l}100 \\
\text { Hours }\end{array}$ & & \multicolumn{10}{|c|}{ Peak Positions $(q)$ and Intensities ( $I)$ (Circular Average) } & \multicolumn{5}{|c|}{ Background-subtracted } \\
\hline & Background & \multicolumn{2}{|c|}{ Perovskite (001) } & \multicolumn{2}{|c|}{$\mathrm{CsPbI}_{3}(002)$} & \multicolumn{2}{|c|}{$\mathrm{FAPbI}_{3}(001)$} & \multicolumn{2}{|c|}{$\mathrm{PbI}_{2}(001)$} & \multicolumn{2}{|c|}{$\mathrm{CsPb}_{3}(102)$} & Perovskite & $\begin{array}{l}\mathrm{CsPbI}_{3} \\
(002)\end{array}$ & $\begin{array}{l}\text { FAPbI }_{3} \\
(001)\end{array}$ & $\begin{array}{l}\mathrm{PbI}_{2} \\
(001)\end{array}$ & $\begin{array}{l}\mathrm{CsPbI}_{3} \\
(102)\end{array}$ \\
\hline & $I$ & $Q$ & $I$ & $Q$ & $I$ & $q$ & $I$ & $Q$ & $I$ & $q$ & $I$ & $I$ & $I$ & $I$ & $I$ & $I$ \\
\hline A & 200.577 & 1.010 & 303.289 & 0.707 & 211.940 & 0.845 & 230.260 & 0.905 & 239.050 & 0.923 & 231.936 & 102.712 & 11.364 & 29.684 & 38.473 & 31.359 \\
\hline B & 197.898 & 1.010 & 284.630 & 0.703 & 210.813 & 0.845 & 229.940 & 0.881 & 239.511 & 0.941 & 232.187 & 86.732 & 12.916 & 32.042 & 41.614 & 34.289 \\
\hline $\mathrm{C}$ & 194.306 & 1.010 & 227.055 & 0.707 & 242.386 & 0.849 & 243.216 & 0.900 & 220.161 & 0.927 & 239.739 & 32.749 & 48.080 & 48.910 & 25.855 & 45.433 \\
\hline $\mathrm{E}$ & 261.700 & 1.014 & 387.500 & 0.707 & 344.843 & 0.849 & 316.700 & 0.900 & 322.400 & 0.923 & 366.800 & 125.800 & 83.143 & 55.000 & 60.700 & 105.100 \\
\hline $\mathrm{E}$ & 179.400 & 1.014 & 276.388 & 0.707 & 192.784 & 0.845 & 206.514 & 0.881 & 217.042 & 0.927 & 208.668 & 96.988 & 13.384 & 27.114 & 37.642 & 29.268 \\
\hline $\mathrm{F}$ & 229.370 & 0.977 & 274.638 & 0.707 & 239.175 & 0.845 & 262.505 & 0.895 & 273.765 & 0.927 & 266.559 & 45.268 & 9.806 & 33.136 & 44.396 & 37.189 \\
\hline
\end{tabular}


Table S6. Peak intensity analysis

\begin{tabular}{|c|c|c|c|c|c|c|c|c|c|}
\hline \multirow[t]{2}{*}{0 Hours } & \multicolumn{5}{|c|}{ Signal/Noise Ratio (If $>1.05$ threshold) $*$} & \multicolumn{4}{|c|}{ Peak Intensity Ratios (if $>0.05$ threshold) $* *$} \\
\hline & Perovskite (001) & $\mathrm{CsPbI}_{3}(002)$ & $\mathrm{FAPbI}_{3}(001)$ & $\mathrm{PbI}_{2}(001)$ & $\mathrm{CsPbI}_{3}(102)$ & $\mathrm{CsPbI}_{3}(002)$ & $\mathrm{FAPbI}_{3}(001)$ & $\mathrm{PbI}_{2}(001)$ & $\mathrm{CsPbI}_{3}(102)$ \\
\hline & $\begin{array}{l}\text { Relative to } \\
\text { background }\end{array}$ & $\begin{array}{l}\text { Relative to } \\
\text { background }\end{array}$ & $\begin{array}{l}\text { Relative to } \\
\text { background }\end{array}$ & $\begin{array}{l}\text { Relative to } \\
\text { background }\end{array}$ & $\begin{array}{l}\text { Relative to } \\
\text { background }\end{array}$ & $\begin{array}{l}\text { Relative to } \\
\text { Perovskite }\end{array}$ & $\begin{array}{l}\text { Relative to } \\
\text { Perovskite }\end{array}$ & $\begin{array}{l}\text { Relative to } \\
\text { Perovskite }\end{array}$ & $\begin{array}{l}\text { Relative to } \\
\text { Perovskite }\end{array}$ \\
\hline A & 10.034 & & 1.130 & 1.497 & & & & 0.055 & \\
\hline B & 8.549 & & 1.077 & 1.726 & & & & 0.096 & \\
\hline $\mathrm{C}$ & 6.552 & 1.731 & 1.111 & 1.409 & 1.596 & 0.132 & & 0.074 & 0.107 \\
\hline $\mathrm{D}$ & 6.863 & 1.580 & & 1.479 & 1.543 & 0.099 & & 0.082 & 0.093 \\
\hline $\mathrm{E}$ & 8.822 & 1.072 & 1.207 & 2.207 & & & & 0.154 & \\
\hline $\mathrm{F}$ & 7.603 & & 1.064 & 2.382 & & & & 0.209 & \\
\hline \multirow[t]{3}{*}{6 Hours } & \multicolumn{5}{|c|}{ Signal/Noise Ratio (If $>0.05$ threshold) } & \multicolumn{4}{|c|}{ Peak Intensity Ratios (if $>0.05$ threshold) } \\
\hline & Perovskite (001) & $\mathrm{CsPbI}_{3}(002)$ & $\mathrm{FAPbI}_{3}(001)$ & $\mathrm{PbI}_{2}(001)$ & $\mathrm{CsPbI}_{3}(102)$ & $\mathrm{CsPbI}_{3}(002)$ & $\mathrm{FAPbI}_{3}(001)$ & $\mathrm{PbI}_{2}(001)$ & $\mathrm{CsPbI}_{3}(102)$ \\
\hline & $\begin{array}{l}\text { Relative to } \\
\text { background }\end{array}$ & $\begin{array}{l}\text { Relative to } \\
\text { background }\end{array}$ & $\begin{array}{l}\text { Relative to } \\
\text { background }\end{array}$ & $\begin{array}{l}\text { Relative to } \\
\text { background }\end{array}$ & $\begin{array}{l}\text { Relative to } \\
\text { background }\end{array}$ & $\begin{array}{l}\text { Relative to } \\
\text { Perovskite }\end{array}$ & $\begin{array}{l}\text { Relative to } \\
\text { Perovskite }\end{array}$ & $\begin{array}{l}\text { Relative to } \\
\text { Perovskite }\end{array}$ & $\begin{array}{l}\text { Relative to } \\
\text { Perovskite }\end{array}$ \\
\hline A & 7.948 & 1.055 & 1.110 & 2.268 & & & & 0.182 & \\
\hline B & 7.627 & 1.101 & 1.133 & 3.673 & & & & 0.403 & \\
\hline $\mathrm{C}$ & 5.447 & 1.703 & 1.100 & 1.238 & 1.517 & 0.158 & & 0.053 & 0.116 \\
\hline $\mathrm{D}$ & 6.230 & 1.647 & 1.099 & 1.838 & 1.559 & 0.124 & & 0.160 & 0.107 \\
\hline$E$ & 7.511 & 1.055 & 1.121 & 2.389 & & & & 0.213 & \\
\hline $\mathrm{F}$ & 7.144 & & 1.128 & 3.919 & & & & 0.475 & \\
\hline
\end{tabular}




\begin{tabular}{|c|c|c|c|c|c|c|c|c|c|}
\hline \multirow[t]{3}{*}{ Hours } & \multicolumn{5}{|c|}{ Signal/Noise Ratio (If $>0.05$ threshold) } & \multicolumn{4}{|c|}{ Peak Intensity Ratios (if $>0.05$ threshold) } \\
\hline & Perovskite (001) & $\mathrm{CsPbI}_{3}(002)$ & $\mathrm{FAPbI}_{3}(001)$ & $\mathrm{PbI}_{2}(001)$ & $\mathrm{CsPbI}_{3}(102)$ & $\mathrm{CsPbI}_{3}(002)$ & $\mathrm{FAPbI}_{3}(001)$ & $\mathrm{PbI}_{2}(001)$ & $\mathrm{CsPbI}_{3}(102)$ \\
\hline & $\begin{array}{l}\text { Relative to } \\
\text { background }\end{array}$ & $\begin{array}{l}\text { Relative to } \\
\text { Perovskite }\end{array}$ & $\begin{array}{l}\text { Relative to } \\
\text { Perovskite }\end{array}$ & $\begin{array}{l}\text { Relative to } \\
\text { Perovskite }\end{array}$ & $\begin{array}{l}\text { Relative to } \\
\text { Perovskite }\end{array}$ & $\begin{array}{l}\text { Relative to } \\
\text { Perovskite }\end{array}$ & $\begin{array}{l}\text { Relative to } \\
\text { Perovskite }\end{array}$ & $\begin{array}{l}\text { Relative to } \\
\text { Perovskite }\end{array}$ & $\begin{array}{l}\text { Relative to } \\
\text { Perovskite }\end{array}$ \\
\hline A & 1.512 & 1.057 & 1.148 & 1.192 & 1.156 & 0.111 & 0.289 & 0.375 & 0.305 \\
\hline B & 1.438 & 1.065 & 1.162 & 1.210 & 1.173 & 0.149 & 0.369 & 0.480 & 0.395 \\
\hline $\mathrm{C}$ & 1.169 & 1.247 & 1.252 & 1.133 & 1.234 & 1.468 & 1.493 & 0.789 & 1.387 \\
\hline $\mathrm{E}$ & 1.481 & 1.318 & 1.210 & 1.232 & 1.402 & 0.661 & 0.437 & 0.483 & 0.835 \\
\hline $\mathrm{E}$ & 1.541 & 1.075 & 1.151 & 1.210 & 1.163 & 0.138 & 0.280 & 0.388 & 0.302 \\
\hline $\mathrm{F}$ & 1.197 & & 1.144 & 1.194 & 1.162 & & 0.732 & 0.981 & 0.822 \\
\hline
\end{tabular}

* Account for systematic error in background subtraction due to amorphous substrates and degradation products (5\%) ** Account for human peak extraction error (5\%) 


\section{References}

1. Correa-Baena, J.-P., Luo, Y., Brenner, T.M., Snaider, J., Sun, S., Li, X., Jensen, M.A., Hartono, N.T.P., Nienhaus, L., Wieghold, S., et al. (2019). Homogenized Halides and Alkali Cation Segregation in Alloyed Organic-inorganic Perovskites. Science 363, 627-631.

2. Menesatti, P., Angelini, C., Pallottino, F., Antonucci, F., Aguzzi, J., and Costa, C. (2012). RGB color calibration for quantitative image analysis: The "3D Thin-Plate Spline" warping approach. Sensors 12, 7063-7079.

3. Stoumpos, C.C., Malliakas, C.D., and Kanatzidis, M.G. (2013). Semiconducting Tin and Lead Iodide Perovskites with Organic Cations: Phase Transitions, High Mobilities, and Near-infrared Photoluminescent Properties. Inorg. Chem. 52, 9019-9038.

4. Zunger, A., Wei, S.H., Ferreira, L.G., and Bernard, J.E. (1990). Special quasirandom structures. Phys. Rev. Lett. 65, 353-356.

5. Van de Walle, A., Asta, M., and Ceder, G. (2002). The alloy theoretic automated toolkit: A user guide. Calphad Comput. Coupling Phase Diagrams Thermochem. 26, 539-553.

6. Van De Walle, A., Tiwary, P., De Jong, M., Olmsted, D.L., Asta, M., Dick, A., Shin, D., Wang, Y., Chen, L.Q., and Liu, Z.K. (2013). Efficient stochastic generation of special quasirandom structures. Calphad Comput. Coupling Phase Diagrams Thermochem. 42, 13-18.

7. Lejaeghere, K., Bihlmayer, G., Björkman, T., Blaha, P., Blügel, S., Blum, V., Caliste, D., Castelli, I.E., Clark, S.J., Dal Corso, A., et al. (2016). Reproducibility in density functional theory calculations of solids. Science 351 .

8. Schelhas, L.T., Li, Z., Christians, J.A., Goyal, A., Kairys, P., Harvey, S.P., Kim, D.H., Stone, K.H., Luther, J.M., Zhu, K., et al. (2019). Insights into operational stability and processing of halide perovskite active layers. Energy Environ. Sci. 12, 1341-1348.

9. SheffieldML/GPyOpt: Gaussian Process Optimization using GPy https://github.com/SheffieldML/GPyOpt.

10. Sollich, P. (2004). Can Gaussian process regression be made robust against model mismatch? In International Workshop on Deterministic and Statistical Methods in Machine Learning (Springer, Berlin, Heidelberg), pp. 199-210. 